\title{
Foundations of Dynamic Monopoly and the Coase Conjecture*
}

\author{
FARUK GUL \\ University of Pennsylvania, Philadelphia, Pennsylvania 19104 \\ HUGO SONNENSCHEIN \\ Princeton University, Princeton, New Jersey 08544 \\ AND \\ ROBERT WILSON \\ Stanford University, Stanford, Califomia 94305 \\ Received June 2, 1985; revised January 15, 1986
}

\begin{abstract}
Subgame-perfect equilibria are characterized for a market in which the seller quotes a price each period. Assume zero costs, positive interest rate, continuum of buyers, and some technical conditions. If buyers' valuations are positive then equilibrium is unique, buyers' strategies are stationary, and the price sequence is determinant along the equilibrium path but possibly randomized elsewhere. Otherwise a continuum of stationary equilibria can exist, but at most one with analytic strategies. Coase's conjecture is verified for stationary strategies: reducing the period length drives all prices to zero or the least valuation. Connections to bargaining models are described. Journal of Economic Literature Classification Number: 022.01986 Academic Press, Inc.
\end{abstract}

\section{INTRODUCTION}

A dynamic theory of monopoly must take into account the fact that a monopolist cannot normally sign contracts to guarantee that the future prices of his output will be above some minimal level. Thus, in a dynamic theory the time path of prices will generally not be the one which, if a com-

* This paper was prepared during Gul and Sonnenschein's visit for a year at the Stanford Business School, which they acknowledge with appreciation. Gul and Sonnenschein acknowledge research support from National Science Foundation grant SES 8510135 to Princeton University; and Wilson, from NSF Grant SES 8308723 and ONR contract N00014-79-C0685 to Stanford University. 
mitment to future prices were possible, would bring forth demands that maximize the discounted stream of revenues minus costs. Let $p_{1}^{c}, p_{2}^{c}, \ldots$ be a maximizing price plan if commitment is possible. Without commitment, after the first price in such a plan, it will almost never be in the monopolist's interest to announce $p_{2}^{c}$. But consumers know this, and so we can expect that they will not anticipate the later prices in the plan when the first price is announced. Thus, even if consumers individually have no market power, they will not purchase in the first period as if the subsequent prices $p_{2}^{c}, p_{3}^{c}, \ldots$ where given. As a consequence, in a dynamic theory it is not in the monopolist's interest to announce $p_{1}^{c}$ in the first period. In order for a plan to be dynamically consistent it must be the case that:

(a) Consumers correctly anticipate prices, and

(b) At every point in time the monopolist can not increase the expected present value of his remaining profit by deviating from the price path that is expected by consumers.

In other words, a dynamic theory of monopoly is an equilibrium theory, and it seems natural that an equilibrium perspective is necessary for analyzing the problem.

To clarify further the necessity for an equilibrium perspective, consider the determination of the first price in a market in which the monopolist announces prices in quick succession: think of a supplier of mineral water standing at his source; assume that he is able to pump at any rate at zero cost and to change his price at will. ${ }^{1}$ Assume also that there are consumers with every valuation less than some arbitrary positive value. On the one hand, one might argue that the monopolist will be able to discriminate perfectly, since the time he needs to make his way down the demand curve will be very short. On the other hand, one might argue to the contrary that the monopolist will make negligible rents: each consumer knows that the monopolist intends to sell eventually to the lowest-valuation consumer, and since the time between offers is short, he believes that the amount of time until the minimal valuation is reached is also short, and thus he will not buy until the price is close to that minimal valuation. The interplay of these factors is the main theme in the recent literature on durable goods monopoly of Bulow [1], Kahn [7], and Stokey [11]. A major result of this paper is to affirm a conjecture of Coase [2] that states that the market will open at a price close to zero. In summary, without repeat purchases monopoly rents must depend substantially on a monopolist's ability to commit to prices or quantities offered in the future.

A second purpose of the paper is to extend Rubinstein's analysis of the

${ }^{1}$ The spirit of the model is best captured by assuming that the water is medicinal, and that only one glass is desired in a lifetime. 
bilateral monopoly bargaining problem with alternating offers to the case that a seller makes repeated offers to many consumers. The striking conclusion of Rubinstein's analysis is that with discounting the bilateral monopoly has a unique subgame-perfect equilibrium in pure strategies, even when there is no a priori restriction on how long the bargaining might continue. In fact, Rubinstein shows that a bargain is reached immediately, with the division of the gains from trade uniquely determined by the parties' rates of discount. That is, discounting is sufficient to render the bargaining problem determinate.

In contrast, we show that the situation in which a monopolist makes repeated offers to a continuum of consumers is considerably more complicated. If the minimum valuation of the consumers exceeds the monopolist's (constant) unit cost, as in Rubinstein's formulation, then again there is generically a unique subgame-perfect equilibrium determined by the distribution of the consumers' valuations, the unit cost, and the discount factor. ${ }^{2}$ This equilibrium predicts a decreasing sequence of prices with sales made in every period until the market is exhausted after a finite number of periods at a final price equal to the least valuation. Off the equilibrium path, however, the monopolist may employ a randomized strategy. In the alternative case that the minimum valuation does not exceed the unit cost, the market remains open forever, and there may be many distinct equilibria. The simple case of a uniform distribution of valuations (i.e., a linear demand function) produces both one equilibrium that involves no randomization off the equilibrium path, and a continuum of equilibria requiring such randomization - and all of these equilibria have different price paths and profits for the monopolist. A substantial regularity assumption, requiring a smooth variation of the consumers' strategies as their valuations vary, is shown to restore the generic uniqueness of the equilibrium. Absent some such assumption, nevertheless, we conclude that in monopolized markets discounting is insufficient in itself to determine the division of the gains from trade. The same lack of uniqueness occurs if the seller makes repeated offers to a single buyer with private information about his valuation. ${ }^{3}$ This suggests a qualitative discontinuity in the equilibria of bargaining problems formulated à la Rubinstein.

The paper is organized as follows: In Section 1 we define the preferences and strategies of the players and explain the notion of equilibrium. In Section 2 we consider a particular example of a market with zero costs of production, and describe its unique equilibrium. The equilibrium exhibits properties that are important in the analysis. First, it requires no randomization along the equilibrium path: prices are determinant and

\footnotetext{
${ }^{2}$ We assume that the monopolist and the consumers all have the same discount factor.

${ }^{3}$ Assume that the seller's cost is interior to the support of the buyer's valuation.
} 
decrease over time. Second, randomization is required off the equilibrium path. Third, the strategies of consumers satisfy a stationarity property; namcly, the distribution of consumers left in the market after any price (that is lower than all preceding prices) is independent of the prior price history in the market. Section 3 states the main existence and uniqueness results for the case that the minimum valuation among the consumers is greater than the unit cost of production, and Section 4 presents the theory for the case that this hypothesis does not hold. Section 5 states the Coase conjecture for arbitrary market demand. Section 6 is composed of a variety of notes, several of which relate our results to existing literature. Among these is the observation that our notion of equilibrium provides foundations for the equilibrium concept used in the theory of durable goods monopoly and that all our theorems apply to that theory. Also, we observe that our existence and uniqueness theorems both generalize and strengthen the work of Sobel and Takahashi [10], Cramton [3], and Fudenberg, Levine, and Tirole [4] on equilibrium for bargaining models in which a seller with known valuation makes price offers to a single consumer whose valuation is a random variable (the value of which is known only to the consumer); such bargaining models have a formal equivalence to the models studied here. The proofs are presented in an Appendix.

\section{SPecification of THE Model}

The monopolist faces a unit (Lebesgue) measure of non-atomic consumers indexed by $q \in[0,1]$. Each consumer is in the market to buy one unit of the monopolist's product, and can buy that unit at any time $i=0,1,2, \ldots$. The preferences of consumers are defined by specifying a nonincreasing left-continuous function $f:[0,1] \rightarrow \mathfrak{R}_{+}$and a discount factor $\delta$. Specifically, if consumer $q \in[0,1]$ buys the product at time $i$ at price $p$, then his utility is $[f(q)-p] \delta^{i 4}$. Assume without loss of generality that $f(q)$ is positive for all $q<1$. At various times, the following two conditions are imposed:

(B) $f(1)$ is positive.

(L) $f$ satisfies a Lipschitz condition at 1 .

The discount factor $\delta$ is positive and less than one; all of the consumers and the monopolist have the same discount factor. ${ }^{5}$ The monopolist's unit costs are constant and zero. ${ }^{6}$ Each consumer maximizes his expected

${ }^{4}$ See 6.7 in Section 6 for the interpretation of the utility function.

${ }^{5}$ Only the coincidence of the consumers' discount factors is necessary for the analysis.

${ }^{6}$ To see that the analysis with zero costs captures the case of a general constant cost $c$, reinterpret prices and consumers' valuations as net of the cost. 
utility, and the monopolist maximizes the expected present value of his revenue stream. ${ }^{7}$

In each period, first the monopolist spccifics a price and then those consumers who have not previously purchased simultaneously choose whether to accept or to reject this price. A consumer who rejects continues as an active player until he eventually accepts some price; his utility is zero if he never accepts an offered price. At any time all players have perfect recall of the previous history of the game.

A strategy for the monopolist specifies at each time a price to charge as a function of the history of the game. ${ }^{8}$ A strategy for a consumer specifies at each time and after each history in which he has not previously purchased whether to accept or to reject the monopolist's offered price; equivalently, it specifies the set of prices the consumer will accept. We seek a subgameperfect Nash equilibrium of this game.

There are some subtle issues involved in defining the game that naturally arise from the above description; for example, technical restrictions are necessary to insure that at each stage the set of consumers accepting an offer is measurable so that the monopolist's revenue can be evaluated. We also argue that in order to characterize the subgame-perfect equilibrium paths of a sensible version of the above game, it is sufficient to consider strategies depending only on the past history of prices.

First, we observe that in this extensive-form game, if the players' strategies prescribe behavior that is optimal for each player for all histories that result from no simultaneous deviations, then the equilibrium path prescribed is the equilibrium path of a subgame-perfect equilibrium. To see this replace that portion of the strategies in any subgame that follows simultaneous deviations by equilibrium behavior in the subgame: this does not change the equilibrium path. Next we assume that the equilibrium actions of each agent are constant on histories in which prices are the same and the sets of agents accepting at each point in time differ at most by sets of measure zero. To some extent this represents a natural regularity requirement; however, the assumption has substantial force and it affects the set of equilibria. It is a maintained hypothesis in the analysis. ${ }^{9}$ With this assumption, unilateral deviations by non-atomic consumers can change neither the actions of the remaining consumers nor the actions of the monopolist. Thus, only unilateral deviations of the monopolist can affect the course of the game. From the observation that simultaneous deviations

\footnotetext{
${ }^{7}$ If $m_{i}$ is the measure of the consumers purchasing in response to the price $p_{i}$ in period $i$ then the monopolist's present value is $\sum_{0}^{\infty} p_{i} m_{i} \delta^{i}$. We adopt the convention that the initial period is $i=0$.

${ }^{8}$ We shall show that the game in which the monopolist chooses quantities is a special case allowing somewhat simpler specification of the off-the-equilibrium-path strategies.

${ }^{9}$ See note 6.1 in Section 6.
} 
from the equilibrium path are unimportant in checking for subgame perfection, it follows that in order to show that a path is associated with a subgame-perfect equilibrium it is necessary and sufficient to specify actions for each agent as functions of the monopolist's previous plays (that is, price histories), so that (a) these functions generate the given path, and (b) after each price history the prescribed actions are optimal.

\section{A Simple Example}

In this example only, the consumers are uniformly distributed on the interval $[0,2]$ with total measure 2 . The monopolist initially holds at least measure 2 of the commodity or can produce at zero cost. Those consumers $q \in[0,1]$ have the valuation 3 and those in $(1,2]$ have the valuation 1 . The discount factor is $\delta=1 / 2$.

There is a unique "perfect foresight" equilibrium, as considered by Bulow [1], Stokey [11], and Kahn [7] for this example. It is given by the price sequence $p_{0}=2, p_{1}=1$, and the sale quantities $m_{0}=m_{1}=1$. These prices and quantities also occur along the equilibrium path in the subgameperfect equilibrium. However, there is no pure strategy equilibrium for the game as specified with the monopolist offering prices.

To see this, first observe that in any equilibrium the prices must be 2 and 1 in the last two periods before sales ccase. If sufficiently few high-valuation consumers remain (less than half as we shall see below), then the monopolist prefers to offer the price 1 and clear the market, so the final price is 1 . Ir the initial price is 1 or the penultimate price exceeds 2 , then all the high-valuation consumers will buy at the final price of 1 (they prefer a price of 1 tomorrow to any price exceeding 2 today); this is not optimal for the monopolist since he can make some sales at a penultimate price not exceeding 2 and do better (as we shall see in more detail below). This price can not be less than 2, however, since if it were then all of the highvaluation consumers would buy (they prefer any price less than 2 to a price of 1 later) and therefore the monopolist prefers to increase any price less than 2: no price between 1 and 2 can be optimal for the monopolist. Thus, the final prices are 2 and then 1 . These can not be the prices in a subgameperfect pure-strategy equilibrium, however. If the monopolist deviates from the prescribed price of 2 and offers a slightly higher price, then either all, some, or none of the high-valuation consumers will purchase. If all, then the next price is expected to be 1 , so their behavior is not optimal. ${ }^{10}$ If some, then the next price is expected to be between 1 and 2 , so that the

\footnotetext{
${ }^{10}$ We ignore here the possibility that a single consumer's choice of whether or not to buy affects the next price charged; see note 6.1 in Section 6.
} 
high-valuation consumers are indifferent about purchasing now rather than waiting; but we have seen that such a price is never optimal for the monopolist. If none, then the next price is expected to be 2 , in which case they should accept the slightly higher price offered now. Thus, there can not be a subgame-perfect equilibrium in pure strategies.

A subgame-perfect equilibrium for this example requires that the monopolist employs a mixed strategy off the equilibrium path. Half of the high-valuation consumers purchase if the price offered does not exceed $2 \frac{1}{2}$, and the other half buy when it does not exceed 2; the low-valuation consumers buy when the price does not exceed 1. If the monopolist charges any price exceeding $2 \frac{1}{7}$, then none of the consumers accept: they expect him to charge 2 next period. If he charges any prices in $\left(2,2 \frac{1}{2}\right]$, then half of the high-valuation consumers accept: they are indifferent about accepting since they expect that next period he will randomize between the prices 2 and 1 (with probabilities that substantiate their indifference). If he charges any price not exceeding 2, then all the high-valuation consumers accept. The monopolist's strategy is to charge 2 if at least half the high-valuation consumers remain, and 1 otherwise - unless he previously deviated by charging a price in $\left(2,2 \frac{1}{2}\right)$, in which case he randomizes between 2 and 1 if precisely half of the high-valuation consumers remain. Note that the randomization following a deviation is optimal for the monopolist since with half of the high-valuation consumers remaining he is indifferent whether to charge 2 now (and 1 next period), or to clear the market by charging 1: both yield a present value of $1 \frac{1}{2}$.

In this example there is no randomization on the equilibrium path, the strategies of consumers are stationary, the equilibrium specifies a determinant decreasing sequence of prices, and the market closes after a finite number of periods. Theorem 1 and its corollary show that we have identified the unique equilibrium for this example, and that the form of the equilibrium is general for markets in which the minimum of the consumers' valuations exceeds the constant unit cost of production.

\section{Markets with Consumers' Valuations BOUNDED AWAY From ZERO}

ThEOREM 1. If $f$ satisfies $(\mathrm{B})$ and $(\mathrm{L})$ then there exist $A \subset[0,1]$, $t:[0,1] \rightarrow[0,1]$, and $P:[0,1] \rightarrow \mathfrak{R}_{++}$such that $\left\{q_{i}, p_{i}\right\}_{i=0}^{\infty}$ is an equilibrium path if and only if $q_{0}=0, q_{1} \in A,(\forall i \geqslant 1) q_{i+1}=t\left(q_{i}\right)$, and $(\forall i \geqslant 0) p_{i}=P\left(q_{i+1}\right){ }^{11}$

"Fudenberg, Levine, and Tirole prove a related result in the context of a bargaining model in which a seller with known valuation makes offers to a consumer whose valuation is a ran- 
Explanation: offer $p_{0}$.

- $q_{1}$ is the initial quantity sold in response to the monopolist's initial

- $t$ determines subsequent quantities sold along the equilibrium path, in terms of the market penetration achieved.

- $P$ defines equilibrium prices as a function of the market penetration achieved.

Let $\Sigma(f, \delta)$ denote the set of equilibria for the market $(f, \delta)$ and let $\Sigma^{s}(f, \delta)$ denote the subset of equilibria which satisfy the condition that the state of the market, after any price that is lower than all preceding prices, is independent of the earlier price history in the market. Equilibria in $\Sigma^{s}(f, \delta)$ are said to be stationary for the consumers, since the sets of those accepting and those rejecting depend only on the current price. The following is an immediate consequence of Theorem 1.

COROLlary. Generic markets satisfying (B) and (L) have a unique equilibrium path and this path leads to a determinate sequence of price offers and acceptances. Furthermore, the path is associated with an equilibrium that is stationary for the consumers, prices are decreasing along the equilibrium path, and all consumers are served after a finite number of offers. ${ }^{12}$

\section{Markets with Valuations Arbitrarily Close to Zero}

Theorem 1 and its corollary are concerned with markets in which the valuations of consumers are bounded aways from zero; that is, assumption (B) is satisfied. For such markets we establish that all equilibria are associated with stationary strategies on the part of the consumers; that is, $\Sigma^{s}(f, \delta)=\Sigma(f, \delta)$. Furthermore, we prove that an equilibrium generically defines a unique decreasing sequence of price offers and acceptances. For markets in which the valuations of consumers are not bounded away from zero, the theory is not nearly so orderly. Before entering into a discussion of these markets, wc would like to make clear why they represent the relevant case.

dom variable. We learned a great deal from their analysis; in particular, our proof of Theorem 1 makes substantial use of their ideas. Our hypotheses are weaker since we do not assume that $f$ is differentiable with differentiable inverse. Also, our conclusion substantially strengtheus the characterization of equilibrium prices. See note 6.2 in Section 6 .

${ }^{12}$ All equilibria are equivalent in the sense that they specify (a) the same equilibrium path, (b) the same strategy for the monopolist, and (c) up to closure, the same acceptance sets for consumers. 
So far discussion has ignored costs of production. This was done because we have in mind stationary constant unit costs, and as we have mentioned (see footnote 6) such costs can be subsumed into the definition of demand (replace $f$ by $f-c$ ). With this formulation prices are interpreted as net of unit cost, and the net valuation of consumers can be negative. Indeed, in a monopolized market, without the possibility for commitment past the current period, the commodity will eventially be sold to all consumers with a net positive valuation. The case in which $f(1)>0$ corresponds to a situation in which there is no "marginal" consumer. When $f(1) \leqslant 0$, the market remains open for an infinite number of periods, and the marginal consumer is identified. Since consumers with negative net valuations are never served, one can consider, without loss of generality, the case $f(1)=0$.

Even when one confines attention to equilibria in $\Sigma^{s}(f, \delta)$, without the assumption (B) that $f(1)>0$ it is not the case that there is a unique equilibrium. In fact, even for the case of linear demand there is a continuum of disjoint equilibrium paths. In Examples 1 and 2 two distinct equilibria are exhibited. In Example 3 it is shown how Example 2 can be altered to produce a continuum of equilibria.

EXAmple 1. We consider the example with a linear demand function $f(q)=1-q$. Stokey [11] studies this example in a Cournot formulation in which the monopolist offers quantities rather than prices. In the equilibrium she derives, in period $i$ after any history that results in sales to the $q_{i}$ consumers with valuations exceeding $1-q_{i}$, the monopolist offers a quantity $\alpha\left[1-q_{i}\right]$ that receives the price $p_{i}=\beta\left[1-q_{i}\right]$, where $\alpha$ and $\beta$ are two parameters to be determined. One can determine $\alpha$ and $\beta$ from symmetry conditions, since along the equilibrium path successive markets are related to each other by a scaling transformation. Thus, if the price is $p_{i}=p\left(q_{i}\right)$ when $q_{i}$ consumers have been served then $p(q)=[1-q] p(0)$ and the monopolist's present value of remaining profits is $R(q)=\frac{1}{2}[1-q]^{2} p(0)$, where the initial price is $p(0)=\beta$ and the initial quantity is $\alpha$. Optimality of the monopolist's strategy requires that $\bar{q}=t(q) \equiv q+\alpha[1-q]$ is the choice that achieves the maximum in the monopolist's associated dynamic programming problem

$$
R(q)=\max _{\bar{q} \geqslant q} P(\bar{q})[\bar{q}-q]+\delta R(\bar{q}),
$$

where $P(\bar{q})$ is the highest price that will induce all consumers with valuations exceeding $f(\bar{q})$ to accept. Utility maximization by the consumers implies that

$$
f(\bar{q})-P(\bar{q})=[f(\bar{q})-P(t(\bar{q}))] \delta,
$$


so that consumer $\bar{q}$ is indifferent between accepting or waiting another period. By hypothesis,

$$
\begin{aligned}
t(\bar{q}) & =\bar{q}+\alpha[1-\bar{q}], \\
P(t(\bar{q})) & =p(\bar{q})=\beta[1-\bar{q}] ;
\end{aligned}
$$

hence,

$$
\begin{aligned}
P(\bar{q}) & =[1-\delta] f(\bar{q})+\delta \beta[1-\bar{q}] \\
& \equiv \gamma[1-\bar{q}],
\end{aligned}
$$

where $\gamma \equiv 1-\delta+\delta \beta$. The unique values that satisfy these relationships are

$$
\alpha=\beta=\bar{\delta} /(1+\bar{\delta}),
$$

where $\bar{\delta} \equiv \sqrt{1-\delta}$. One can further verify using the methods developed later that with these values a subgame-perfect equilibrium is in fact obtaincd. The Coase conjecture is verified in this example by noting that as $\delta \rightarrow 1$ the initial price $p(0)=\beta \rightarrow 0$, the initial quantity $\alpha \rightarrow 0$, and the monopolist's present value $R(0) \rightarrow 0$. Also, if one interprets the increase in the discount factor as due to a shortening of the duration of a period, say $\delta \equiv e^{-r \Delta}$ and $\Delta \rightarrow 0$, then the limiting value of each consumer's expected utility is his valuation; that is, trades occur early.

EXAMPLE 2. In this example we assume the same linear demand function $f(q)=1-q$ as in Example 1, but we require that the discount factor is sufficiently large. We construct an equilibrium with strikingly different properties, although the equilibrium path has a superficial resemblance to the equilibrium path of Example 1 and it enjoys the same asymptotic properties as $\delta \rightarrow 1$. In each period $i$ after serving $q_{i}$ consumers the monopolist charges the price $p_{i}=\beta\left[1-q_{i}\right]$ and sells the quantity $\alpha\left[1-q_{i}\right]$; thus, $\beta=p_{0} /\left[1-q_{0}\right]$ and $\alpha=\left[q_{1}-q_{0}\right] /\left[1-q_{0}\right]$, or starting from $q_{0}=0$ the initial price is $p_{0}=\beta$ and the initial quantity is $q_{1}=\alpha$. Similarly,

$$
\begin{aligned}
t\left(q_{i}\right) & =q_{i}+\alpha\left[1-q_{i}\right], \\
p_{i} & =[1-\alpha]^{i} p_{0}, \\
P\left(q_{i}\right) & =[1-\delta]\left[1-q_{i}\right]+\delta p_{i},
\end{aligned}
$$

along the equilibrium path, precisely as along the equilibrium path of Examplc 1. The rescmblance ends here, however, since the equilibrium values of $\alpha$ and $\beta$ are different, and the strategies off the equilibrium path are quite different. 
The key to the construction of this equilibrium is the specification of the strategies off the equilibrium path. The form of the equilibrium strategies is the following: In any period (not necessarily period $i$ ), if those consumers previously served are those with valuations exceeding $1-q$ and $q \in\left(q_{i-1}, q_{i}\right)$, then the monopolist charges $p_{i}$. He does the same if $q=q_{i}$ unless in the previous period he deviated and charged a price $p \in\left(\hat{p}_{i}, p_{i-1}\right)$, where $\hat{p}_{i-1} \equiv[1-\delta]\left[1-q_{i-1}\right]+\delta p_{i}$, in which case he randomizes between the prices $p_{i}$ and $p_{i+1}$ with probabilities determined so as to make the consumer with valuation $1-q_{i}$ indifferent whether to accept the price $p$ in the previous period or to wait for the subsequent lottery between the next two prices. The consumers' responses have essentially the simple form derived in Example 1: one with the valuation $1-q$ accepts any price $p \leqslant P(q)$, where if $q \in\left(q_{i-1}, q_{i}\right]$ then

$$
P(q)=[1-\delta][1-q]+\delta p_{i} ;
$$

note, however, that unlike Example 1 in this case the consumers' reservation price strategy is represented by the piecewise-linear left-continuous decreasing function $P$ with downward jumps at $q_{i}$ of magnitude $\delta\left[p_{i}-p_{i+1}\right]$.

An equilibrium of this form entails the following relationships: First, the present value of the monopolist's subsequent revenues after serving the $q$ consumers with valuations exceeding $1-q$ is piecewisc-lincar and continuous of the form $R(q)=R_{i}+p_{i}\left[q_{i}-q\right]$ if $q \in\left(q_{i-1}, q_{i}\right]$ and $R_{i} \equiv R\left(q_{i}\right)$. In addition,

$$
\begin{aligned}
& p_{i}=[1-\delta]\left[1-q_{i+1}\right]+\delta p_{i+1}, \\
& R_{i}=p_{i}\left[q_{i+1}-q_{i}\right]+\delta R_{i+1}, \\
& R_{i}=R_{i+1}+p_{i+1}\left[q_{i+1}-q_{i}\right]
\end{aligned}
$$

which express respectively the consumers' behavior, the recursion for the monopolist's present value, and the continuity of the monopolist's present value function, all along the equilibrium path. An immediate consequence of (2) and (3) is that

$$
R_{i}=p_{i+1}\left[q_{i+2}-q_{i}\right]+\delta R_{i+2},
$$

which with (2) assures that the monopolist is in fact willing to randomize at $q_{i}$ between the prices $p_{i}$ and $p_{i+1}$ when required after a previous deviant price $p \in\left(\hat{p}_{i}, p_{i-1}\right)$. The conditions (1), (2), and (3) have a solution

$$
p_{i}=\bar{\alpha}^{i} p_{0}, \quad R_{i}=\bar{\alpha}^{2 i} R_{0}, \quad 1-q_{i}=\bar{\alpha}^{i}\left[1-q_{0}\right]
$$


where

$$
p_{0}=\left[1-q_{0}\right] \frac{\bar{\alpha}-\delta \bar{\alpha}}{1-\delta \bar{\alpha}}, \quad R_{0}=p_{0}\left[1-q_{0}\right] \frac{\bar{\alpha}}{1+\bar{\alpha}},
$$

and $\bar{\alpha}$ is determined as the solution in $(0,1)$ of the equation ${ }^{13}$

$$
\bar{\alpha}=\frac{1-\bar{\alpha}^{2}}{1-\delta \bar{\alpha}^{2}} \text {. }
$$

One can show that $\bar{\alpha} \rightarrow 1$ as $\delta \rightarrow 1$; indeed, as a function of $\delta, \bar{\alpha}$ is convex and increasing with an infinite rate of increase at 1 . In terms of the specification above,

$$
\alpha=1-\bar{\alpha} \quad \text { and } \quad \beta=\frac{\bar{\alpha}-\delta \bar{\alpha}}{1-\delta \bar{\alpha}},
$$

which both tend to 0 as $\delta \rightarrow 1$.

The verification that this specification yields an equilibrium can be accomplished in two parts. For the first part we can apply the following lemma, which is a consequence of repeated applications of (2) and (3): If $j \geqslant i+2$ then $R_{i}>p_{j}\left[q_{j+1}-q_{i}\right]+\delta R_{j+1}$. Along the equilibrium path this assures that the monopolist prefers to name the price $p_{i}$ at $q_{i}$ rather than any price $p_{j}<p_{i+1}<p_{i}$. For the second part we must verify that at any $q \in\left(q_{i-1}, q_{i}\right]$ the monopolist prefers the price $p_{i}$ to any other $p \neq p_{i}$ (except $p_{i+1}$ if $q=q_{i}$ ). We omit the lengthy derivation of this result except to remark that the proof depends on the assumption that $\bar{\alpha}^{2}>\frac{1}{2}$, which is assured if $\delta>2-\sqrt{2}$. Thus if the discount factor is sufficiently large then the specification yields an equilibrium.

EXAMPLE 3. We now turn to the demonstration that the equilibrium derived in Example 2 can be generalized to generate a continuum of equilibria. The key observation is to note that in the construction of Example 2 the specification of $q_{0}$ is a free parameter. For each sufficiently small negative value of $q_{0}$ there exists an additional equilibrium in which the play of the game proceeds as follows: The monopolist begins with $q=0$ which lies in one of the intervals $\left(q_{j-1}, q_{j}\right]$ generated by the choice of $q_{0}$. Interpret this situation as the initiation of a subgame imbedded in the larger game corresponding to the choice of $q_{0}$; that is, imagine that the measure 1 of consumers present is the residual after a portion $\left|q_{0}\right|$ of a larger population of measure $1-q_{0}$ has been served. Then, the equilibrium prescribes that the monopolist opens with the initial offer $p_{j}$, and that the consumers

${ }^{13}$ A typical example with $q_{0}=0$ and $\delta=0,9$ yields $\bar{\alpha}=0.8247, p_{0}=0.3199$ and $R_{0}=0.1446$. 
with valuations no less than $1-q_{j+1}$ accept. Similarly, if the monopolist deviates and opens with any offer $p \in\left(p_{j}, p_{j-1}\right]$ then those consumers with valuations no less than $1-q_{j}$ accept, and in particular if $p \in\left(\hat{p}_{j}, p_{j-1}\right)$ then they expect that next time he will randomize between the prices $p_{j}$ and $p_{j+1}$. And, if he opens with a price exceeding $p_{j-1}$ then no consumers accept. All these behaviors are simply the subgame-perfect equilibrium strategies in the subgame of the game in which the 'real' game is interpreted as imbedded. After these opening moves, the play continues precisely in the same fashion, using the critical values $q_{k}$ and $p_{k}$ for $k \geqslant j$ generated from the choice of $q_{0}$.

From this construction, therefore, we see that the market with a linear demand function and a discount factor sufficiently large has a continuum of equilibria. All of these equilibria have entirely disjoint equilibrium paths, though they share many features in common such as described above: in each case the monopolist's prices in successive periods have a constant ratio, and after the first period a constant percentage of the unserved consumers accept each period (these constants differ between Example 1 and Examples 2 and 3).

The possibility of a continuum of equilibria presents scrious difficultics for the theory. The striking feature of Rubinstein's [8] seminal paper on the bargaining problem is that it demonstrates that even with an infinite horizon, impatience is sufficient to give a determinant solution to the bargaining problem. Theorem 1 tells us that this conclusion remains true with one-sided offers and many consumers when the valuation of the monopolist is not a member of the set of valuations of the consumers. The preceding examples tell us that when one leaves such a regime, one loses the determinacy of the solution. In a noncooperative game with a continuum of players and a continuum of equilibria, it is difficult to invoke an argument to select among the equilibria, to judge any one more likely than another, or even to rest assured that the players' expectations will enable any equilibrium to be realized. Thus, whether or not the valuation of the monopolist is disjoint from the set of consumers' valuations represents a critical distinction for the theory.

Observe that among the many equilibria for the linear demand case there is only one (Example 1) for which $P$, the function specifying the consumers' strategies, is continuous. This suggests the following conjecture: if the demand function $f$ is continuous then there exists a unique equilibrium $\sigma \in \Sigma^{s}(f, \delta)$ such that the associated function $P$ is continuous in some neighborhood of 1 . We argue that such an equilibrium is a salient predictor of market behavior, for two reasons. First, where $P$ is continuous the equilibrium specifies a pure strategy for the seller off the equilibrium path (as well as on the path). Second, general considerations of continuity 
indicate that this class of equilibrium selections is the only plausible candidate that could ensure that small changes in the data of the problem (e.g., variations in $f$ ) inducc correspondingly small changes in the agents' strategies. Unfortunately we have not been able to establish this conjecture in the strong form mentioned; instead, we establish uniqueness of the equilibrium for which $P$ is analytic in a neighborhood of 1 , using the following construction. Suppose that $P$ and $P^{*}$ specify equilibrium strategies for the consumers in the market $(f, \delta)$, where $f$ has an $n$th order derivative at 1 . We show that if the derivatives of $n$th order at 1 also exist for $P$ and $P^{*}$, then these derivatives are identical. This is then shown to imply that if $f$ has continuous derivatives of all orders at 1 , and $P$ and $P^{*}$ are analytic at 1 , then $P$ and $P^{*}$ are identical functions. For instance, this result confirms that in the case of linear demand the equilibrium path constructed by Stokey [11], as in Example 1, is the only one sustained by an analytic strategy for the consumers.

THEOREM 2. Assume that $f(1)=0, n \geqslant 1, f \in C^{n}(1)$, and $f^{\prime}(1) \neq 0$. Consider two equilibria, $\sigma_{P}, \sigma_{Q} \in \Sigma^{s}(f, \delta)$ for which $P, Q:[0,1] \rightarrow \mathfrak{R}_{+}$specify the stationary strategies of the consumers, and for $k \leqslant n$ let $P^{k}$ and $Q^{k}$ denote their $k$ th order derivatives. Then, if $P^{k}(1)$ and $Q^{k}(1)$ exist they are equal. Moreover, if $(\forall n) f \in C^{n}(1)$, and $P$ and $Q$ are analytic in a neighborhood of 1 , then $(\forall q \in(0,1]) P(q)=Q(q)$.

Note. It is easy to show that if $P$ is analytic in a neighborhood of 1 , then for any $\delta$ and any $n$ there exists a demand function $f \in C^{n}(1)$ so that $P$ defines an equilibrium of the market $(f, \delta)$. The equilibrium so defined will be in $\Sigma^{s}(f, \delta)$. This is one way to see that the analyticity assumption does not render the problem vacuous.

\section{The CoAse Conjecture}

Finally, we resolve the Coase conjecture [2] with a general result that requires only that the consumers' strategies are stationary. ${ }^{14}$

THEOREM 3 (Coase conjecture). For each $\varepsilon>0$ there exists $\bar{\delta}<1$ such that for all $\delta>\bar{\delta}$ and for all equilibria $\sigma \in \Sigma^{s}(f, \delta)$, the first price prescribed by $\sigma$ is less than $\varepsilon$.

${ }^{14}$ The theorem is stated for the case that $f(1)=0$. If $f(1) \neq 0$, then the statement need not include the hypothesis that $\sigma \in \Sigma^{x}(f, \delta)$ and the first price prescribed by $\sigma$ will be less than $f(1)+\varepsilon$. The proof of this case is less difficult and follows the ideas in the first half of the proof of Theorem 3 . 
Since each consumer has the option of accepting the first price offered, we obtain the following corollary:

COROLLARY. For each $\varepsilon>0$ there exists $\bar{\delta}<1$ such that for all $\delta>\bar{\delta}$ and for all equilibria $\sigma \in \Sigma^{s}(f, \delta)$, a consumer $q$ with the valuation $f(q)$ obtains an equilibrium payoff not less than $f(q)-\varepsilon$.

The proof of Theorem 3 in the Appendix is rather complicated, so here we sketch a more intuitive line of argument, although it is incomplete in several respects. The key consideration is that, since the consumers' equilibrium strategies are stationary, the monopolist has the option at any time to accelerate the process by offering tomorrow's price today and thereby advancing the acceptance dates of subsequent consumers. The cost of doing this is the foregone higher profit on those consumers accepting today, whereas the benefit is the interest on the monopolist's present value of continuation, which is thereby made to arrive a day earlier. Since an equilibrium requires that exercising this option must be disadvantageous for the monopolist, we know that the cost must exceed the benefit. But the cost is approximately the price cut times the number of consumers who accept today's price, and the benefit is the daily interest on the continuation value. Consequently, the daily interest on the continuation vaiue is bounded by approximately the day-to-day price drop times the number accepting per day. Fix the interest rate per unit time to be $100 \%$, and divide this inequality through twice by the length of a day: then the continuation value divided by the length of a day is bounded by the product of the rates (per unit time) at which prices decline and consumers accept. As the length of a day shrinks, the rate of price decline must be bounded or consumers would prefer to wait rather than accept the current price. If the rate of acceptance is also bounded, then as the length of a day shrinks the continuation value must also shrink to zero-if opportunities remain for the monopolist to reduce his price. If the continuation value shrinks to zero then the monopolist's later prices must all be converging to his unit cost, and therefore his present prices too: otherwise, if the day is sufficiently short then the consumers all prefer to delay purchasing. If no opportunities for further price reductions remain then the price must already be at its minimum, which is the minimal valuation among the consumers. The remaining case, therefore, is that the rate of acceptances is unbounded. But in this case also the prices offered by the monopolist must all be converging downward to his unit cost (or the consumers' least valuation), since this is the only way that a positive fraction of the consumers will accept in each of several days when their interest cost of delay is small; that is, the sequence of prices must become flat in the limit, yet the sequence is tied down at the end. In outline, this is one interpretation of the arguments supporting the Coase conjecture. 


\section{NoTES}

6.1. We demonstrate that a genuine restriction is imposed by the assumption that agents treat as equivalent those histories that differ only by the actions of sets of consumers of measure zero. We do so by showing that for a slightly altered version of the example in Section 2, there is an equilibrium in which the monopolist distinguishes among "equivalent" histories and that has a different equilibrium path than obtains if he can not make such distinctions.

Alter the example in Section 2 so that the consumers with the valuation 3 have measure 2 (rather than 1). It remains true that there is an equilibrium in which the sequence of prices is first 2 and then 1 . A second equilibrium that distinguishes among equivalent histories has a different equilibrium path, as follows: Consider the consumers' strategies specified by the function

$$
P(q)=\left\{\begin{array}{lll}
2 \frac{1}{2} & \text { if } & q \in\left[0, \frac{3}{2}\right], \\
2 & \text { if } & q \in\left(\frac{3}{2}, 2\right], \\
1 & \text { if } & q \in(2,3] .
\end{array}\right.
$$

Suppose that the monopolist charges $2 \frac{1}{2}$ first and then charges 1 provided that all consumers $q \leqslant \frac{3}{2}$ accept the first offer. If one or more of these consumers to not accept the first offer, and no other agent does accept, then the monopolist next charges 2 followed by the final offer of 1 . Observe that the consumers $q \leqslant \frac{3}{2}$ can do no better than to accept the first offer of $2 \frac{1}{2}$ since their expectations of the subsequent price depend on whether or not each one accepts. Thus, with an off-the-equilibrium-path strategy for the monopolist specified similarly to the original example, this provides an alternative equilibrium with a different equilibrium path.

6.2. The formalism of Theorems $1-3$ and their corollaries accommodates the case of bilateral bargaining in which a seller with a known valuation repeatedly makes offers to a single buyer with a privately known valuation whose probability distribution is common knowledge. If $F$ is the cumulative distribution function (assumed invertible for simplicity), then the buyer of type $q$ has the valuation $f(q)=F^{-1}(1-q)$; that is, the rightcumulative distribution functions is interpreted as the inverse demand function. The appropriate criterion for the bargaining problem is a sequential equilibrium. For a subgame-perfect equilibrium of the monopoly problem, given any price history the residual demand in the monopoly market defines the seller's posterior distribution of the buyer's type in the corresponding sequential equilibrium of the bargaining problem after the same price history. It is for this reason that analyses of bargaining models 
with repeated offers by the uninformed party have produced results formally identical to those obtained in analyses of durable goods monopoly; see for example Fudenberg, Levine, and Tirole [4] on the one hand and (for the case of linear demand) Stokey [11] on the other. This equivalence is surprising, since the histories in the monopoly market include the particular sets of consumers who have purchased at each price, whereas in the bargaining problem the buyer says only "no" until he accepts and the game terminates. In fact, our previous note indicates that these two games are not formally identical in the absence of our maintained hypothesis that agents do not distinguish among histories differing only by the actions of consumers (or types of the buyer) of measure zero. In particular, the alternative equilibrium described in $\mathbf{6 . 1}$ is not a sequential equilibrium for the corresponding bargaining model in which a single seller with valuation 0 makes offers to a buyer who is twice as likely to have the valuation 3 as 1 and both parties use the discount factor $\delta=\frac{1}{2}$. Only with the hypothesis that agents can not distinguish among equivalent histories do the two models become formally identical.

With the formalism of this paper interpreted as applying to the bargaining problem, Theorem 1 and its corollary strengthen a theorem of Fudenberg, Levine, and Tirole [4] in two ways. First, we dispense with their assumption that the cumulative distribution function of the buyer's valuation is differentiable and has a differentiable inverse. More significantly, we show that there is no randomization along the equilibrium path, so that (generically) there is a determinate sequence of price offers. ${ }^{15}$

Section 4 can also be interpreted as applying to the bargaining problem, with the added possibility that the buyer's valuation may be no more than the seller's. In this case exchange may never occur if there are no gains from trade. Previous analysis of this problem did not discover the equilibria in Examples 2 and $3 .{ }^{16}$ The existence of multiple equilibria suggests a qualitative discontinuity as the supports of the buyer's and the seller's valuations intersect; this discontinuity is also likely to appear in bargaining with alternating offers. ${ }^{17}$

For the case that assumption (B) is satisfied, Theorem 1 proves both existence and uniqueness of equilibrium. Fudenberg, Levine, and Tirole [4] provide an existence theorem for markets in which the demand function does not satisfy assumption (B). The idea of their proof is to consider the limit of a sequence of equilibria for markets satisfying (B), with demand functions $f(\cdot)+b$ as $b$ decreases to zero. The proof that a limit

\footnotetext{
${ }^{15}$ Fudenberg, Levine, and Tirole [4] allow a random path of price offers, with each price depending on the realizations of earlier price randomizations.

${ }^{16}$ See, for example, Sobel and Takahashi [10] and Cramton [3].

${ }^{17}$ See, for example, Rubinstein [9], Cramton [3], and Grossman and Perry [5].
} 
exists and is an equilibrium at $b=0$ is rather intricate and we have not verified that it would apply to the more general class of demand functions that we admit. Combined with Theorem 1, however, application of their method may lead to a generalization of their existence theorem.

6.3. Stokey [11] analyzes a model of durable-good monopoly with perfect secondary markets using a rational expectations formulation. The monopolist chooses a profit maximizing sequence of cumulative quantities offered. Deviations from the profit-maximizing plan are important in her analysis (hence, her use of the term "perfection"), but her model is not game-theoretic since neither the preferences nor the actions of the consumcrs arc modeled explicitly. Nevertheless, her model and ours specify the same equilibrium path. In addition to providing game-theoretic foundations for her specification, our results can be interpreted as clarifying the general problem of existence and uniqueness of equilibria for her model. Stokey focuses on the Coase conjecture and the case of linear demand. She verifies the conjecture for the special case of the equilibrium presented in Example 1.

6.4. One can define an analog of our model in which the monopolist chooses quantities rather than prices, and which leads to the same equilibrium path of quantities and prices as in our model. To do so in a complete game-theoretic formulation requires a specification of how prices are detcrmincd when a sequence of quantities is offered on the market. This is accomplished by adopting an auction procedure. This formulation leads to rather complicated strategies for the buyers, however: stationarity is lost since each consumer's bids change over time.

6.5. Kahn [7] introduces quadratic production costs into Stokey's model with linear demand and considers the case that, as the period length shrinks, the cost functions converges to the continuous-time total cost function

$$
\gamma[Q(\cdot)]=c \int_{0}^{\infty}\left[Q^{\prime}(t)\right]^{2} e^{-r t} d t
$$

where $Q$ is a path of cumulative production. For the discrete-time model he identifies an equilibrium similar to Example 1 (that is, the monopolist serves a fraction of the remaining consumers that is invariant with respect to the history). For this equilibrium, he observes that as the period length shrinks the monopolist's production path does not converge to the efficient path; and in fact yields positive profit, thus excluding an analog of the Coase conjecture. Kahn's result reinforces our theme that monopoly rents depend on the monopolist's ability to commit to future prices or sales for some duration. Increasing costs, and hence the necessity of spreading 
production over time, enable the monopolist to commit credibly to constrain the rate of supply offered in the near future. Kenneth Arrow has suggested to us that decreasing costs may also provide means for credible commitments.

6.6. Gul $\lfloor 6\rfloor$ studies the problem of dynamic oligopoly. He proves that with two or more firms the perfection requirement on the seller's strategies (a strategy must be profit maximizing after every history) imposes no restriction on the total profits that can be earned in equilibrium. This refutes the analog of the Coase conjecture for oligopolistic markets; moreover, he shows that there is no tendency towards the perfectly competitive outcome as the number of firms is increased or the period length shrinks.

6.7. We offer two interpretations of the utility functions of the consumers. In the first, a consumer $q$ receives $f(q)$ "utiles" at the instant he consumes the product, and he has use for at most one unit. Utiles are measured so that at any time $\$ 1$ provides a flow of utility having a present value of 1 utile. Thus, if consumer $q$ purchases in period $i$ at the price $p_{i}$ then he obtains the utility $f(q) \delta^{i}$ and gives up $p_{i} \delta^{i}$. The consumer maximizes utility by timing his purchase to make the expectation of $\left[f(q)-p_{i}\right] \delta^{i}$ as large as possible. In the second interpretation he obtains $[1-\delta] f(q)$ utiles per period in each period after purchase, whereas one unit of the numeraire commodity (money) gives each consumer $1-\delta$ utiles each period. Note that the value of one dollar held for one period is $[1-\delta] / \delta$ tomorrow or $\delta[(1-\delta) / \delta]=1-\delta$ today. Thus, a consumer who in period $i$ trades $p_{i}$ of the numeraire for a unit of the durable commodity changes his utility according to the value of the stream

$$
[1-\delta]\left\{0,0, \ldots, f(q)-p_{i}, f(q)-p_{i}, \ldots\right\},
$$

where the first nonzero element is in period $i$. The value of this stream is $\left[f(q)-p_{i}\right] \delta^{i}$ and this accounts for the form of the utility function.

The absence of infusions of new demand into the market is central to our analysis. However, the model does not require that consumers purchase only one unit. The demand function can just as well be viewed as the integral of the demand functions of consumers. As a very special case, each consumer could have the same demand function $f$; in this situation the mean demand is also $f$.

Finally we observe that none of our results depend substantially on the assumption that the monopolist has the same time preferences as the consumers. On the other hand, our methods do not apply to the case that consumers' discount factors differ. 
6.8. For the case that $f(1)=0$, the possibility of non-stationary equilibria follows from the existence of multiple stationary equilibria such as we exhibited in Example 3. Let $\sigma$ and $\sigma^{\prime}$ be two equilibria in $\Sigma^{s}(f, \delta)$, using $P$ and $P^{\prime}$ to define the stationary strategies of the consumers in the two equilibria. Assume that $P \neq P^{\prime}$ and that the monopolist's profit is not less in $\sigma$ than in $\sigma^{\prime}$. Let $p_{0}$ be the monopolist's initial offer using $\sigma$, and consider the following strategy: If the monopolist charges $p_{0}$ initially then $\sigma$ is followed thereafter; otherwise $\sigma^{\prime}$ is followed; finally specify that the monopolist does charge $p_{0}$ initially. Clearly this is an equilibrium strategy but is not a member of $\Sigma^{s}(f, \delta)$, since each consumer's strategy depends on the initial price offered. Recalling from Example 3 that a continuum of equilibria is possible, it is evident that this approach enables the construction of highly nonstationary equilibria in which at every time the selection of the continuation depends on the entire history of prices.

\section{APPENDIX: PROOFS}

Note. In all of the following "equilibrium" means "subgame-perfect equilibrium." Assumptions (B) and (L) are assumed in Theorem 1 and its preceding lemmas.

LEMMA 1. In any equilibrium $\sigma$ and after any history, if the state in period $i$ is $q_{i}$, then the present value of the monopolist's expected profit is at least $\left[1-q_{i}\right] f(1)$; that is

$$
R^{\sigma}\left(q_{i}\right) \geqslant\left[1-q_{i}\right] f(1),
$$

and the monopolist's price prescribed by $\sigma$ is at least $f(1)$.

Proof. It is sufficient to observe that in equilibrium all of the consumers accept the price $f(1)$. Suppose this were not so, and for any selected equilibrium let $c \leqslant f(1)$ be the supremum of the prices that will be accepted by all consumers (except possibly for a set of measure zero) after any history. If $c=f(1)$ and a positive measure of consumers reject $c$ then no optimal strategy exists for the monopolist, so assume that $c<f(1)$. An optimal strategy for the monopolist can not specify an offer less than $c$, since any such offer is less than an offer that is sure to be accepted by all remaining consumers. Consider the offer $p=[1-\delta] f(1)+\delta c$. By construction, each consumer prefers the offer $p-\varepsilon$ now to an anticipated offer $c$ later. But notice that $p-\varepsilon>c$ for small $\varepsilon>0$ since $c<f(1)$. Therefore, $p-\varepsilon$ will be accepted now by every consumer. Since this is true for every date and history, the definition of $c$ as a supremum is contradicted. Thus we conclude that $c \geqslant f(1)$. 
Lemva 2. There exists $q<1$ such that in any equilibrium and in any period $i$ after any history, if the state is $q_{i} \geqslant q$ and the next periods state is $q_{i+1}$ when the actions prescribed by the equilibrium strategies are taken, then $q_{i+1}=1$.

Proof. Since the function $f$ is Lipschitzian at 1 , there exists $q^{*}<1$ and $k$ such that if $\bar{q}>q^{*}$ then

$$
f(\bar{q}) \leqslant f(1)+k[1-\bar{q}] .
$$

Given any equilibrium $\sigma$ and any history, if $q_{i}$ is the state and $p_{i}$ is the price prescribed by $\sigma$, then the state $q_{i+1}$ in the next period will satisfy $q_{i} \leqslant q_{i+1} \leqslant 1$, and since $f$ is left-continuous and consumer $q$ does not purchase at price $p_{i}$ if $p_{i}>f(q)$,

$$
f\left(q_{i+1}\right) \geqslant p_{i} .
$$

Since every consumer remaining in the market in period $i+1$ at state $q_{i+1}$ has a valuation not exceeding $f\left(q_{i+1}\right)$, we obtain

$$
R^{\sigma}\left(q_{i}\right) \leqslant\left[q_{i+1}-q_{i}\right] p_{i}+\delta\left[1-q_{i+1}\right] f\left(q_{i+1}\right) .
$$

By Lemma 1 and Eqs. (3), (2), and then (1) above, if $1 \geqslant q_{i+1} \geqslant q_{i}>q^{*}$ then

$$
\begin{aligned}
0 \geqslant & {\left[1-q_{i}\right] f(1)-R^{\sigma}\left(q_{i}\right) } \\
\geqslant & {\left[1-q_{i}\right] f(1)-\left[q_{i+1}-q_{i}\right] f\left(q_{i+1}\right)-\delta\left[1-q_{i+1}\right] f\left(q_{i+1}\right) } \\
\geqslant & {\left[1-q_{i}\right] f(1)-\left[q_{i+1}-q_{i}\right]\left\{f(1)+k\left[1-q_{i+1}\right]\right\} } \\
& -\delta\left[1-q_{i+1}\right]\left\{f(1)+k\left[1-q_{i+1}\right]\right\} \\
\geqslant & {[1-\delta]\left[1-q_{i+1}\right] f(1)-\left[q_{i+1}-q_{i}\right]\left[1-q_{i+1}\right] k-\delta\left[1-q_{i+1}\right]^{2} k } \\
\geqslant & {\left[1-q_{i+1}\right]\left\{[1-\delta] f(1)-\left[1-q_{i}\right] k-\delta\left[1-q_{i}\right] k\right\} . }
\end{aligned}
$$

The term in the last bracket is positive for all sufficiently small values of $1-q_{i}$. Therefore, there exists $q<1$ such that $q_{i} \geqslant q$ implies that $1-q_{i+1}=0$.

Definition. A pair $(q, P)$ is a reservation price strategy if it satisfies the following three properties:

(i) $0 \leqslant q<1$, and $P:[q, 1] \rightarrow \Re_{++}$is non-increasing and left-continuous. 
In any equilibrium and in any period $i$ after any history, if $q_{i}$ is the state and $\bar{q}>q$, and if the monopolist offers any price $p$ in period $i$, then

(ii) if $p<P(\bar{q})$ then $q_{i+1} \geqslant \bar{q}$, and

(iii) if $p>P(\bar{q})$ then $q_{i+1}<\bar{q}$.

LEMma 3. There exists a reservation price strategy pair $(q, P)$.

Proof. Define $P(\bar{q}) \equiv[1-\delta] f(\bar{q})+\delta f(1)$ for all $\bar{q} \in[q, 1]$, where $q$ is defined as in the statement of Lemma 2. Obviously $P$ satisfies (i). Assume that $p_{i}<P(\bar{q})$ and $q_{i+1}<\bar{q}$; then consumer $\bar{q}$ does not buy in period $i$. The greatest utility that $\bar{q}$ can obtain is bounded by

$$
\begin{aligned}
{[f(\bar{q})-f(1)] \delta^{i+1} } & =[f(\bar{q})-(1-\delta) f(\bar{q})-\delta f(1)] \delta^{i} \\
& =[f(\bar{q})-P(\bar{q})] \delta^{i} \\
& <\left[f(\bar{q})-p_{i}\right] \delta^{i} ;
\end{aligned}
$$

hence, $\bar{q}$ should purchase the good in period $i$, which contradicts utility maximization. Similarly, if $p_{i}>P(\bar{q})$ and $q_{i+1} \geqslant \bar{q}$ then from the fact that $q_{i+1} \geqslant \bar{q}>q$ and from Lemma 2 , we know that $q_{i+1}=1$. Thus, $p_{i+1}$ will be $f(1)$. As before,

$$
[f(\bar{q})-f(1)] \delta^{i+1}=[f(\bar{q})-P(\bar{q})] \delta^{i},
$$

so

$$
\left[f(\bar{q})-p_{i}\right] \delta^{i}<[f(\bar{q})-f(1)] \delta^{i+1} .
$$

Since $f$ is left-continuous, the above inequality also holds for some $q^{\prime}<\bar{q}$, and so by utility maximization the consumer $q^{\prime}$ must not buy in period $i$. This contradicts the fact that $q_{i+1} \geqslant \bar{q}$.

Definition. Fix a reservation price strategy pair $(r, P)$ and define

$$
L\left(\bar{Q},\left\{Q_{j}, \pi_{j}\right\}_{j=0}^{\infty}\right) \equiv \sum_{j=0}^{\infty} \pi_{j}\left[Q_{j+1}-Q_{j}\right] \delta^{j},
$$

and specify the constrained maximization problem:

$$
Z(\bar{Q}) \equiv \max _{\mathscr{C}} L\left(\bar{Q},\left\{Q_{j}, \pi_{j}\right\}_{j=0}^{\infty}\right)
$$

where $\mathscr{C}$ is the set of sequences $\left\{Q_{j}, \pi_{j}\right\}_{j=0}^{\infty}$ satisfying the constraints

$$
Q_{0}=\bar{Q}, \quad r \leqslant Q_{1}, \quad Q_{j} \leqslant Q_{j+1} \leqslant 1, \quad \pi_{j} \leqslant P\left(Q_{i+1}\right) .
$$


Also, define

$$
M(\bar{Q}) \equiv\{Q \mid P(Q)[Q-\bar{Q}]+\delta Z(Q)=Z(\bar{Q})\}
$$

LEMMA 4. For any reservation price strategy pair $(r, P)$ the constrained maximization problem $[(\mathrm{A})$ subject to $(\mathrm{C})]$ has a solution and any solution has the property that $(\forall j) \pi_{j}=P\left(Q_{j+1}\right)$ and $Q_{j+1}>Q_{j}$ (or $Q_{j}=Q_{j+1}=1$ ). Further, there exists $r^{\prime}<r\left(\right.$ or $\left.r^{\prime}=r=0\right)$ such that in any equilibrium and in any state $q_{i} \geqslant r^{\prime}$ in any period $i$ after any history,

$$
R^{\sigma}\left(q_{i}\right)=Z\left(q_{i}\right)
$$

The set of solutions has the properties that

$$
\inf M(\bar{Q}) \in M(\bar{Q}) \quad \text { and } \quad Q^{\prime}>Q \in M(Q) \Rightarrow P\left(Q^{\prime}\right)<P(Q) \text {. }
$$

If $q_{i}=\bar{Q} \geqslant r^{\prime}$ then the next state is $q_{i+1} \in M(\bar{Q})$. The function

$$
\hat{P}(\bar{Q}) \equiv P(\inf M(\bar{Q}))
$$

is non-increasing and left-continuous; in particular, if $Q>\bar{Q}$ then

$$
p^{1} \in P(M(\bar{Q})) \quad \text { and } \quad p^{2} \in P(M(Q)) \Rightarrow p^{1} \geqslant p^{2} .
$$

Proof. That (A) has a solution follows from the fact that $P$ is left-continuous and non-increasing. That $\pi_{j}=P\left(Q_{j+1}\right)$ and (if $Q_{j}<1$ ) that $Q_{j+1}>Q_{j}$ are obvious.

We first establish (i). Let $\left\{Q_{j}, \pi_{j}\right\}_{j=0}^{\infty}$ be a solution to (A) for $\bar{Q}=q_{i}$. Suppose that $R^{\sigma}\left(q_{i}\right)=Z\left(q_{i}\right)-\varepsilon$ for some $\varepsilon>0$, and set $p_{i+j}=\pi_{j}-\varepsilon / 2$. If the monopolist follows the strategy $\left\{p_{i+j}\right\}_{j=0}^{\infty}$ after period $i$, then the present value of his profit is at least

$$
\sum_{j=0}^{\infty} p_{i+j}\left[Q_{j+1}-Q_{j}\right] \delta^{j}=Z\left(q_{i}\right)-\left[1-q_{i}\right] \varepsilon / 2>R^{\sigma}\left(q_{i}\right)
$$

which contradicts the optimality of the monopolist's plan. Hence, $R^{\sigma}\left(q_{i}\right) \geqslant Z\left(q_{i}\right)$ for all $q_{i} \in[0,1]$. On the other hand, if $q_{i} \geqslant r$ then the $p$ 's and $q$ 's specified by the equilibrium strategy $\sigma$ are feasible for (A); hence, also $R^{\sigma}\left(q_{i}\right) \leqslant Z\left(q_{i}\right)$. We will now use the fact that if $q_{i}<r$ and $q_{i+1}<r$ then

$$
R^{\sigma}\left(q_{i}\right)<\left[r-q_{i}\right] f(0)+\delta Z(r) .
$$

For $q_{i}$ sufficiently close to $r$,

$$
\left[r-q_{i}\right] f(0)+\delta Z(r)<Z(r),
$$


so

$$
R^{\sigma}\left(q_{i}\right)<Z(r) \leqslant Z\left(q_{i}\right),
$$

which is a contradiction. Thus, if $q_{i}$ is sufficiently close to $r$ then $q_{i+1} \geqslant r$; and hence, once again the $p$ 's and $q$ 's specified by $\sigma$ are feasible for (A), implying that $R^{\sigma}\left(q_{i}\right) \leqslant Z\left(q_{i}\right)$, which proves the desired result.

To show that $m \equiv \inf M(\bar{Q}) \in M(\bar{Q})$, we begin with the observation that $M(\bar{Q})$ is bounded and that $m \geqslant r$. Let $\left\{x_{t}\right\}_{t-0}^{\infty} \subset M(\bar{Q})$ be a decreasing sequence converging to $m$. Since $P$ is left-continuous and non-increasing, $p^{*} \equiv \lim P\left(x_{i}\right) \leqslant P(m)$. From the definition of $M$,

$$
Z(\bar{Q})=P\left(x_{t}\right)\left[x_{t}-\bar{Q}\right]+\delta Z\left(x_{t}\right),
$$

for all $t$. Since $Z$ is continuous,

$$
Z(\bar{Q})=p^{*}[m-\bar{Q}]+\delta Z(m) .
$$

If $p^{*}<P(m)$ then

$$
Z(\bar{Q})<P(m)[m-\bar{Q}]+\delta Z(m),
$$

which contradicts the definition of $Z(\bar{Q})$ and the principle of optimality of dynamic programming. Hence $P(m)=p^{*}$, implying that $m \in M(\bar{Q})$.

If $Q^{\prime}>Q \in M(\bar{Q})$ then it is obvious that $P(Q)>P\left(Q^{\prime}\right)$. Furthermore, note that $\bar{Q}=q_{i}>r^{\prime}$ implies that $q_{i+1} \in M(\bar{Q})$, using $R^{\sigma}\left(q_{i}\right)=Z\left(q_{i}\right)$ as established earlier and the principle of optimality.

Finally, wc establish (iii). If $p^{1} \in P(M(\bar{Q})), p^{2} \in P(M(Q)), Q>\bar{Q}$, and $p^{1}<p^{2}$, then there exist $x_{1} \in M(\bar{Q})$ and $x_{2} \in M(Q)$ such that $P\left(x_{1}\right)<P\left(x_{2}\right)$. (Since $P$ is non-increasing this implies that $x_{1}>x_{2}$.) Therefore,

$$
\begin{aligned}
Z(\bar{Q}) & \geqslant P\left(x_{2}\right)\left[x_{2}-\bar{Q}\right]+\delta Z\left(x_{2}\right) \\
& =P\left(x_{2}\right)[Q-\bar{Q}]+P\left(x_{2}\right)\left[x_{2}-Q\right]+\delta Z\left(x_{2}\right) \\
& =P\left(x_{2}\right)[Q-\bar{Q}]+\delta Z(Q),
\end{aligned}
$$

Similarly,

$$
Z(Q) \geqslant-P\left(x_{1}\right)[Q-\bar{Q}]+\delta Z(\bar{Q})
$$

so

$$
0 \geqslant\left[P\left(x_{2}\right)-P\left(x_{1}\right)\right][Q-\bar{Q}],
$$

hence

$$
P\left(x_{1}\right) \geqslant P\left(x_{2}\right)
$$


which is a contradiction. Thus we have established that $p^{1} \in P(M(\bar{Q}))$, $p^{2} \in P(M(Q))$, and $Q>\bar{Q}$ imply that $p^{1} \geqslant p^{2}$. Since inf $M(\bar{Q}) \in M(\bar{Q})$ and inf $M(Q) \in M(Q)$ we have $\hat{P}(\bar{Q}) \in P(M(\bar{Q}))$ and $\hat{P}(Q) \in P(M(Q))$. Using the result stated in the previous sentence, $Q>\bar{Q}$ implies $\hat{P}(\bar{Q}) \geqslant \hat{P}(Q)$; that is, $\hat{P}$ is non-increasing.

Now let $\left\{x_{i}\right\}_{l=0}^{\infty}$ be an increasing sequence converging to $\bar{Q}$ and define

$$
p^{*} \equiv \lim _{t \rightarrow \infty} \hat{P}\left(x_{t}\right)
$$

The limit exists since $\hat{P}$ is non-increasing. Define $y_{t} \equiv \inf M\left(x_{t}\right)$ for all $t$. Then $\left\{y_{t}\right\}$ has a convergent subsequence and without loss of generality assume that $\left\{y_{t}\right\}$ converges to $y$. Since $\hat{P}$ is non-increasing we have $p^{*} \leqslant P(\bar{Q}) ;$ also,

$$
Z\left(x_{t}\right)=P\left(y_{t}\right)\left[y_{t}-\bar{Q}\right]+\delta Z\left(y_{t}\right),
$$

for all $t$. Since $Z$ is continuous,

$$
Z(\bar{Q})=p^{*}[y-\bar{Q}]+\delta Z(y) .
$$

Since $P$ is non-increasing and left-continuous we have $p^{*} \leqslant P(y)$. If $p^{*}<P(y)$ then substituting $P(y)$ into the preceding equality contradicts the defining property of $Z$, so $p^{*}=P(y)$. Thus, $y \in M(\bar{Q})$ and therefore $\hat{P}(\bar{Q}) \leqslant P(y)=p^{*}$, proving that $p^{*}=\hat{P}(\bar{Q})$ and establishing the left-continuity of $\hat{P}$.

Notation. In the following we let $\hat{P}(\cdot ;(r, P))$ and $M(\cdot ;(r, P))$ be the functions $\hat{P}$ and $M$ as defined in Lemma 4 using $(r, P)$ as the reservation price strategy pair.

LEMMA 5. If $(q, P)$ is a reservation price strategy pair satisfying the consumer-equilibrium property ${ }^{18}$

$$
(\forall \ddot{q} \geqslant q) \quad P(\bar{q})=[1-\delta] f(\bar{q})+\delta \hat{P}(\bar{q} ;(q, P))
$$

and $q>0$, then there exists a reservation price strategy pair $\left(q^{\prime}, P^{\prime}\right)$ with $q^{\prime}<q$ that also satisfies $(\mathrm{CF})$ for which $P^{\prime}(\bar{q})=P(\bar{q})$ for all $\bar{q} \geqslant q$.

Proof. For all $\bar{q} \in[0,1]$ define

$$
P^{\prime}(\bar{q}) \equiv[1-\delta] f(\bar{q})+\delta \hat{P}(\bar{q} ;(q, P)) .
$$

Note that $P^{\prime}$ is left-continuous and non-increasing. Furthermore, since $(q, P)$ satisfies (CE) we know that $P^{\prime}(\bar{q})=P(\bar{q})$ whenever $\bar{q} \geqslant q$.

${ }^{18}$ See the definition that follows Lemma 2. 
We now show that there exists $q^{\prime}<q$ such that $\left(q^{\prime}, P^{\prime}\right)$ satisfies (CE) Fact (ii) of Lemma 4 establishes the existence of $q^{\prime}<q$ such that in any equilibrium $\sigma$ and after any history, if the state $q_{i} \geqslant q^{\prime}$ and $q_{i}=\bar{q}$ ther $q_{i+1} \in M(\bar{q} ;(q, P))$, where $q_{i+1}$ is the state in period $i+1$. Observe that the argument used in proving this result establishes that $M(\bar{q} ;(q, P))=$ $M\left(\bar{q} ;\left(q^{\prime}, P^{\prime}\right)\right)$ for all $\bar{q} \geqslant q^{\prime}$, so also

$$
\inf M(\bar{q} ;(q, P))=\inf M\left(\bar{q} ;\left(q^{\prime}, P^{\prime}\right)\right)
$$

for all $\bar{q} \geqslant q^{\prime}$. By definition, inf $M(\bar{q} ;(q, P)) \geqslant q$; hence,

$$
\begin{aligned}
\hat{P}\left(\bar{q} ;\left(q^{\prime}, P^{\prime}\right)\right) & =P^{\prime}\left(\inf M\left(\bar{q} ;\left(q^{\prime}, P^{\prime}\right)\right)\right) \\
& =P(\inf M(\bar{q} ;(q, P)))=\hat{P}(\bar{q} ;(q, P)),
\end{aligned}
$$

and so

$$
P^{\prime}(\bar{q})=[1-\delta] f(\bar{q})+\delta \hat{P}\left(\bar{q} ;\left(q^{\prime}, P^{\prime}\right)\right)
$$

for all $\bar{q} \geqslant q^{\prime}$. Therefore $\left(q^{\prime}, P^{\prime}\right)$ satisfies (CE).

Next we prove that $\left(q^{\prime}, P^{\prime}\right)$ satisfies (ii) of Lemma 3; that is, we show that $q_{i}<\bar{q}, \bar{q} \geqslant q^{\prime}$, and $p_{i}<P^{\prime}(\bar{q})$ imply that $q_{i+1} \geqslant \bar{q}$. If $\bar{q} \geqslant q$ then, since $P^{\prime}(\bar{q})=P(\bar{q})$, the fact that $(q, P)$ satisfies (ii) implies that also $\left(q^{\prime}, P^{\prime}\right)$ satisfies (ii). Now suppose that $\bar{q}<q$. By the definition of $q^{\prime}$, if $q_{i+1} \geqslant q^{\prime}$ then $q_{i+2} \in M\left(q_{i-1} ;(q, P)\right)$ and hence $q_{i+2} \geqslant q>q$. If $\bar{q}>q_{i+1}$ then $q_{i+2}>\bar{q}>q_{i+1}$; that is, consumer $\bar{q}$ buys in period $\mathrm{i}+1$. But, if $\sigma$ prescribes $p_{i+1}$ then by Lemma 4 ,

$$
p_{i+1}=P\left(q_{i+2}\right) \in P\left(M\left(q_{i+1}\right) ;(q, P)\right) .
$$

Recall that $\hat{P}(\bar{q} ;(q, P)) \in P(M(\bar{q}) ;(q, P))$ and $\bar{q}>q_{i+1}$ so by (iii) of Lemma $4, p_{i+1} \geqslant \hat{P}(\bar{q} ;(q, P))$. However by the definition of $P^{\prime}$,

$$
\left[f(\bar{q})-P^{\prime}(\bar{q})\right] \delta^{i}=[f(\bar{q})-\hat{P}(\bar{q} ;(q, P))] \delta^{i+1},
$$

so

$$
\left[f(q)-p_{i}\right] \delta^{i}>\left[f(\bar{q})-p_{i+1}\right] \delta^{i+1}
$$

Thus, consumer $q$ prefers buying in period $i$ to buying in period $i+1$, contradicting utility maximization. (For the case that $\bar{q}<q$ and $q_{i+1}<q^{\prime}$, (ii) is established by considering $p_{k}$, where $q_{k}<\bar{q}$ and $q_{k+1} \geqslant \bar{q}$.)

Finally, we show that $\left(q^{\prime}, P^{\prime}\right)$ satisfies (iii) of Lemma 4. Assume that $q_{i}<\bar{q}, \bar{q}>q^{\prime}, p_{i}>P(\bar{q})$, and $q_{i+1} \geqslant q^{\prime}$. If $\bar{q} \geqslant q$ then, since $P^{\prime}(\bar{q})=P(\bar{q})$, the fact that $(q, P)$ satisfies (iii) yields the result that $\left(q^{\prime}, P^{\prime}\right)$ also satisfies (iii). 
Suppose, therefore, that $\bar{q}<q$ and thus $q_{i+1} \geqslant \bar{q}>q_{i}$. Reasoning as above we obtain

$$
\left[f(\bar{q})-p_{i}\right] \delta^{i}<\left[f(\bar{q})-p_{i+1}\right] \delta^{i+1} .
$$

Since $f$ is left-continuous we can find $q^{*} \in\left(q_{i}, \bar{q}\right)$ for which the above inequality also holds: that is consumer $q^{*}$ would rather buy in period $i+1$ than in period $i$. But $q_{i}<q^{*}<q_{i+1}$ means that $q^{*}$ buys in period $i$, contradicting utility maximization.

Theorem 1. If $f$ satisfies (B) and (L) then there exist $A \subset[0,1]$, $t:[0,1] \rightarrow[0,1]$, and $P:[0,1] \rightarrow \Re_{++}$such that $\left\{q_{i}, p_{i}\right\}_{i=0}^{\infty}$ is an equilibrium path if and only if $q_{0}=0, q_{1} \in A,(\forall i \geqslant 1) q_{i+1}=t\left(q_{i}\right)$, and $(\forall i \geqslant 0) p_{i}=P\left(q_{i+1}\right)$.

Proof. First note that Lemma 3 assures the existence of a reservation price strategy pair $(q, P)$. Lemma 5 establishes that any such pair can be extended to a larger interval domain (smaller $q$ ) and retain the same properties, including condition (CE); furthermore, it is clear that the lower limit of such domains is not bounded away from zero. Hence, one such pair has $q=0$ and satisfies (CE). Any other reservation price strategy pair $\left(0, P^{\prime}\right)$ has $P^{\prime}(\bar{q})=P(\bar{q})$ for all $\bar{q} \in(0,1)$. Set $A \equiv M(0)$ and $(\forall \bar{q} \in[0,1])$ $t(\bar{q}) \equiv \inf M(\bar{q})$. We claim that $(A, t, P)$ has the properties required by Theorem 1 .

First we prove the "only if" part of the statement. Let $\sigma$ be any equilibrium. By (i) in Lemma $4, R^{\sigma}(0)=Z(0)$, and so $q_{1} \in M(0)=A$. Also by Lemma $4, p_{0}=P\left(q_{1}\right)$. We next show that $p_{i+1}=P\left(t\left(q_{i+1}\right)\right)$ for all $i \geqslant 0$. By Lemma 4 we have $R^{\sigma}\left(q_{i}\right)=Z\left(q_{i}\right), q_{i+1} \in M\left(q_{i}\right), p_{i}=P\left(q_{i+1}\right)$, and $q_{i+1}>q_{i}$ (or $\left.q_{i}=1\right)$ for all $i \geqslant 0$. Since $(0, P)$ satisfies (CE) we have

$$
\left[f\left(q_{i+1}\right)-p_{i}\right] \delta^{i}=\left[f\left(q_{i+1}\right)-\hat{P}\left(q_{i+1}\right)\right] \delta^{i+1},
$$

and since an interval of consumers purchase in each period, the left continuity of $f$ and utility maximization by the consumers imply that $p_{i+1} \geqslant \hat{P}\left(q_{i+1}\right)$-otherwise some consumer $\bar{q}$ would not purchase in period $i$. But $\hat{P}\left(q_{i+1}\right)=P\left(\inf M\left(q_{i+1}\right)\right)$ and $P$ is non-increasing, so $\hat{P}\left(q_{i+1}\right)$ is the largest optimal price in period $i+1$. Thus, $p_{i+1} \leqslant \hat{P}\left(q_{i+1}\right)$, which proves that $p_{i+1}=\hat{P}\left(q_{i+1}\right)=P\left(t\left(q_{i+1}\right)\right)$.

Finally, observe that if $x=\inf M\left(q_{i+1}\right) \in M\left(q_{i+1}\right)$ then $q_{i+2} \geqslant x$, since $q_{i+2} \in M\left(q_{i+1}\right)$. But

$$
P(x)=P\left(t\left(q_{i+1}\right)\right)=p_{i+1}=P\left(q_{i+2}\right) .
$$

Hence, if $q_{i+2}>x$ then

$$
x \in M\left(q_{t+1}\right), \quad q_{t+2}>x, \quad \text { and } \quad P\left(q_{i+2}\right)=P(x),
$$


which contradicts (ii) of Lemma 4. Therefore

$$
q_{i+2}=x=\inf M\left(q_{i+1}\right)=t\left(q_{i+1}\right),
$$

which completes the proof of the "only if" part of the statement.

To prove the "if" part of the statement, choose $q_{1} \in M(0)$ and $p_{0}=P\left(q_{1}\right)$, and define strategies $\sigma$ as follows:

The consumers. After any history, if the state is $q_{i}$ then consumer $\bar{q} \in\left(q_{i}, 1\right]$ buys if and only if $p_{i} \leqslant P(\bar{q})$.

The monopolist. After any history, if the state is $q_{i}$ then $p_{i}=P\left(t\left(q_{i}\right)\right)$ if $p_{i-1} \geqslant P\left(q_{i}\right)$, and otherwise $p_{i}$ is chosen randomly to be either $\hat{P}\left(q_{i}\right)$ with probability $\beta$ or $p_{l}$ with probability $1-\beta$ where

$$
\begin{aligned}
& p_{l}=\lim _{q \downarrow q_{i}} \hat{P}(q), \\
& x=\lim _{q \downarrow q_{i}} f(q), \\
& \beta= \begin{cases}1 & \text { if } x-p_{i-1} \leqslant\left[x-\hat{P}\left(q_{i}\right)\right] \delta \\
\beta\left(p_{i-1}\right) & \text { otherwise, }\end{cases}
\end{aligned}
$$

and $\beta(p)$ is the solution to

$$
x-p=\beta\left[x-\hat{P}\left(q_{i}\right)\right] \delta+(1-\beta)\left[x-p_{l}\right] \delta .
$$

First we note that $\beta \in[0,1]$. If $\beta \neq 1$ then

$$
x-p_{i-1} \geqslant\left[x-\hat{P}\left(q_{i}\right)\right] \delta ;
$$

hence it suffices to show that

$$
x-p_{i-1} \leqslant\left[x-p_{l}\right] \delta .
$$

Recall that, for all $q$,

$$
f(q)-P(q)=[f(q)-\hat{P}(q)] \delta,
$$

and therefore,

$$
\lim _{q \downarrow q_{i}}[f(q)-P(q)]=\lim _{q \downarrow q_{i}}[f(q)-\hat{P}(q)] \delta
$$

and

$$
x-\lim _{q \downarrow q_{i}} P(q)=\left[x-p_{l}\right] \delta .
$$

But $p_{i-1} \geqslant \lim _{q \downarrow \eta_{i}} P(q)$, so we have established the desired result. 
Next we observe that after any history resulting in a state $q_{i}$ in period $i$, the monopolist charges either $\hat{P}\left(q_{i}\right), p_{l}$, or a mixture of the two. The optimality of $\hat{P}\left(q_{i}\right)$ follows from its definition and Lemma 4 . To prove the optimality of $p_{l}$ we first note that $\inf M(q)$ is a non-decreasing function - using the argument made in proving that $\hat{P}$ is non-increasing. Let $\left\{x_{t}\right\}$ be a decreasing sequence converging $q_{i}$ and define $y_{t} \equiv \inf M\left(x_{t}\right)$ and $y=\lim y_{t}$. Then

$$
p_{l}=\lim P\left(y_{i}\right) \leqslant P(y)
$$

and

$$
Z\left(x_{t}\right)=P\left(y_{t}\right)\left[y_{t}-x_{t}\right]+\delta \angle\left(y_{t}\right)
$$

which, since $Z$ is continuous, implies that

$$
Z\left(q_{i}\right)=p_{i}\left[y-q_{i}\right]+\delta Z(y) .
$$

Thus $P(y) \leqslant p_{l}$, proving that $P(y)=p_{l}$. This shows that $p_{l}$ is an optimal price in state $q_{i}$. Since $\hat{P}\left(q_{i}\right)$ and $p_{t}$ are optimal, every randomization between them is also optimal. This establishes that the specified strategy for the monopolist is optimal.

To prove optimality of the consumers' strategy we first show that the consumers never regret not purchasing when their strategy prescribes not to purchase. Consider any history resulting in a state $q_{i}$ in period $i$, and a price $p_{i}$ offered by the monopolist. If $p_{i}>P(\bar{q})$ for some $\bar{q} \in\left(q_{i}, 1\right]$ then $q_{i+1}<\bar{q}$ and the (possibly random) price $\tilde{p}_{i+1}$ that will follow is such that it makes consumer $q_{i+1}$ indifferent between buying in period $i$ or period $i+1$. Hence,

$$
\left[f\left(q_{i+1}\right)-p_{i}\right] \delta^{i}=\mathbf{E}\left\{f\left(q_{i+1}\right)-\tilde{p}_{i+1}\right\} \delta^{i+1}
$$

so

$$
\left[f\left(q_{i+1}\right)-p_{i}\right] \delta^{i}=\left[f\left(q_{i+1}\right)-\mathbf{E}\left\{\tilde{p}_{i+1}\right\}\right] \delta^{i+1}
$$

implying

$$
\left[f(\bar{q})-p_{i}\right] \delta^{i}=\left[f(\bar{q})-\mathbf{E}\left\{\tilde{p}_{i+1}\right\}\right] \delta^{i+1} .
$$

Thus consumer $\vec{q}$ does not regret not buying in period $i$, since he can do at least as well by waiting an additional period.

Finally we prove that consumers do not regret purchasing whenever the strategy $\sigma$ prescribes that they purchase. After any history resulting in state $q_{i}$ in period $i$ the strategy $\sigma$ prescribes that consumer $\bar{q} \in\left(q_{i}, 1\right]$ buys if and only if the offered price $p_{i}$ satisfies $p_{i} \leqslant P(\bar{q})$. Assume for the moment that, 
according to $\sigma, p_{i}$ is followed by a sequence of non-random prices $p_{j}$ and states $q_{j}, j>i$. In this case,

$$
\left[f\left(q_{j+1}\right)-p_{j}\right] \delta^{j}=\left[f\left(q_{j+1}\right)-p_{j+1}\right] \delta^{j+1},
$$

for all $j \geqslant i$. Since $q_{j+1} \geqslant \bar{q}$ for all $j \geqslant i$,

$$
f(\bar{q})-p_{i} \geqslant\left[f(\bar{q})-p_{j+1}\right] \delta \quad(\forall j \geqslant i) .
$$

It follows that

$$
\left[f(\bar{q})-p_{i}\right] \delta^{i} \geqslant\left[f(\bar{q})-p_{j+1}\right] \delta^{j+1} \quad(\forall j \geqslant i),
$$

which establishes the required result. On the other hand, if $p_{i}$ is followed by a nondegenerate random variable $\tilde{p}_{i+1}$, then

$$
\left[f\left(q_{i+1}\right)-p_{i}\right] \delta^{i}=\left[f\left(q_{i+1}\right)-\mathbf{E}\left\{\tilde{p}_{i+1}\right\}\right] \delta^{i+1},
$$

and $q_{i+1} \geqslant \bar{q}$. Hence, consumer $\bar{q}$ likes buying in period $i$ at least as much as waiting for the next period; and repeating the reasoning above he weakly prefers any outcome of the randomization to any price that follows. This establishes the optimality of buying in period $i$ for consumer $\bar{q}$ and completes the proof.

THEOREM 2. Assume that $f(1)=0, n \geqslant 1, f \in C^{n}(1)$, and $f^{\prime}(1) \neq 0$. Consider two equilibria $\sigma_{P}, \sigma_{Q} \in \Sigma^{s}(f, \delta)$ for which $P, Q:[0,1] \rightarrow \Re_{+}$specify the stationary strategies of the consumers, and for $k \leqslant n$ let $P^{k}$ and $Q^{k}$ denote their $k$ th order derivatives. Then, if $P^{k}(1)$ and $Q^{k}(1)$ exist they are equal. Moreover, if $(\forall n) f \in C^{n}(1)$, and $P$ and $Q$ are analytic in a neighborhood of 1 , then $(\forall q \in(0,1]) P(q)=Q(q)$.

Proof. Recall that $f(q) \geqslant P(q) \geqslant[1-\delta] f(q)$ and $f(1)=0$; consequently $P(1)=0$, and since $f^{\prime}(1) \neq 0, \quad P^{\prime}(1) \neq 0$. Choose $\varepsilon>0$ such that $P \in C^{n}([1-\varepsilon, 1])$. Define $Z(q)$ for $q \in[1-\varepsilon, 1]$ as in Lemma 4 of Theorem 1, and define

$$
\bar{Z}\left(t_{0}, q\right)=P\left(t_{0}\right)\left[t_{0}-q\right]+\delta Z\left(t_{0}\right)
$$

for all $q \in[1-\varepsilon, 1]$ and $t_{0} \in[q, 1]$. Since $P$ is continuously differentiable and $P^{\prime}(1) \neq 0$, for $q$ sufficiently close to $1, \bar{Z}$ is strictly concave in $t_{0}$. Hence the function

$$
t(q)=\arg \max _{t_{0} \in[q, 1]} \bar{Z}\left(t_{0}, q\right)
$$

is well defined and $Z^{\prime}(q)=-P(t(q))$ by a standard result in dynamic programming. The argument used in proving Lemma 4 of Theorem 1 
establishes that after any $i$-period history resulting in state $q_{i}$, if $q_{i}$ is sufficiently close to 1 then the price in period $i$ will be $P\left(t\left(q_{i}\right)\right)$. Hence, $t$ defines the strategy of the monopolist for states sufficiently close to 1 . Therefore, for such $q$ we have by consumer optimality

$$
P(q)-[1-\delta] f(q)+\delta P(t(q)) .
$$

Since $P \in C^{1}(1)$, profit maximization implies that

$$
d \bar{Z} / d t_{0} \equiv P^{\prime}(t(q))[t(q)-q]+P(t(q))+\delta Z^{\prime}(t(q))=0
$$

for $q$ close to 1 . Substituting expressions from above yields

$$
P^{\prime}(t(q))[t(q)-q]+[1-\delta] f(t(q))=0 .
$$

Since $P^{\prime}(1) \neq 0$, applying the implicit function theorem to Eq. (1) yields that $t$ has the same order of differentiability as $P$; that is, $t \in C^{n}(1)$. Hence Eq. (2) implies, using l'Hôpital's rule, that

$$
P^{\prime}(1)=-\frac{[1-\delta] f^{\prime}(1) t^{\prime}(1)}{t^{\prime}(1)-1}
$$

Differentiating Eq. (1) implicitly, evaluating it at $q=1$, and using Eq. (3) yields

$$
t^{\prime}(1)=(1-\sqrt{1-\delta}) / \delta \quad \text { and } \quad P^{\prime}(1)=f^{\prime}(1) \sqrt{1-\delta} .
$$

Repeating the argument for $Q$ and the corresponding $s$ associated with $Q$ yields the same conclusion; therefore $P^{\prime}(1)=Q^{\prime}(1)$ and $t^{\prime}(1)=s^{\prime}(1)$. To complete the proof of the first part of the theorem it suffices to show that $P^{k}(1)=Q^{k}(1)$ and $t^{k}(1)=s^{k}(1)$ for all $k \leqslant n-1$ implies that $P^{n}(1)=Q^{n}(1)$ and $t^{n}(1)=s^{n}(1)$. Observe that Eq. (2) implies, for $q$ close to 1 , that

$$
\begin{aligned}
& P^{\prime}(t(q))[t(q)-q]+[1-\delta] f(t(q))-Q^{\prime}(s(q))[s(q)-q] \\
& -[1-\delta] f(s(q))=0 .
\end{aligned}
$$

Differentiating Eq. (4) $n-1$ times, dividing the result by $t(q)-q$, and taking the limit as $q \rightarrow 1$, one obtains a linear equation in the variables $\left[Q^{n}(1)-P^{n}(1)\right]$ and $\left[s^{n}(1)-t^{n}(1)\right]$. Also, from Eq. (1) we have

$$
P(q)-\delta P(t(q))-Q(q)+\delta Q(s(q))=0 .
$$

Differentiating Eq. (5) $n$ times and evaluating the result at 1 we obtain a second linear equation. This pair of equations forms a homogeneous linear system, and the determinant of the matrix of coefficients depends only on 
the first derivatives of $f, P$, at 1 , and is nonzero for all $n$. This implies that $P^{n}(1)=Q^{n}(1)$ and $t^{n}(1)=s^{n}(1)$, as desired to complete the induction.

To prove the second part of the theorem, observe that if $f^{\prime}(1) \neq 0$ and $f \in C^{n}(1)$ for all $n$ then by the previous argument all derivatives of $P$ and $Q$ at 1 are equal. If $P$ and $Q$ are analytic in some neighborhood of 1 then, for some $\varepsilon>0, P(q)=Q(q)$ for all $q \in[1-\varepsilon, 1]$. Following the result from Theorem 1 that $P$ can be extended uniquely from such an interval to the interval $(0,1]$ yields that $P(q)=Q(q)$ for all $q \in(0,1]$, as required.

THEOREM 3 (Coase conjecture). For each $\varepsilon>0$ there exists $\bar{\delta}<1$ such that for all $\delta>\delta$ and for all equilibria $\sigma \in \Sigma^{s}(f, \delta)$, the first price prescribed by $\sigma$ is less than $\varepsilon$.

Proof. Let $0, r_{1}, r_{2}, \ldots$ be an ordering of the rational 2 in the interval $[0,1]$. If the theorem is false then there exists $\varepsilon>0$ such that for all $\bar{\Delta}>0$ there exists $\Delta<\bar{\Delta}$ and $P_{\Delta}$ such that $P_{\Delta}(0)>\varepsilon$. Here, $P_{\Delta}$ is the $P$-function associated with an equilibrium in $\Sigma^{s}(f, \delta)$. Consider any sequence $\Delta_{n} \rightarrow 0$ such that $P_{\Delta_{n}}(0)>\varepsilon$ for all $n=1,2, \ldots$. Select a convergent subsequence $\left\{P_{\Delta_{j}}(0)\right\}$ and define $P(0) \equiv \lim _{j \rightarrow \infty} P_{d_{j}}(0)$. Now from the sequence $\left\{P_{\Delta_{j}}\left(r_{1}\right)\right\}$ select a convergent subsequence with a limit defined to be $\bar{P}\left(r_{1}\right)$. Continue in this fashion to define $\bar{P}$ on all the rationals $\mathscr{Q}$ in $[0,1]$. Extend $\bar{P}$ to the entire interval by imposing left-continuity. Note that from the procedure used to construct $\bar{P}$ and the fact that $P_{A_{n}}$ is non-increasing for each $n$, it follows that also $\bar{P}$ is non-increasing. In the following, let $\delta=e^{-r \Delta}$.

We first show that assuming the function $\bar{P}$ so constructed is continuous yields a contradiction. Thus, suppose that $\bar{P}$ is continuous. Then there exist $x_{1}$ and $x_{2}>x_{1}$ such that

$$
\bar{P}(0)>\bar{P}\left(x_{1}\right) \geqslant \bar{P}\left(x_{2}\right)>0,
$$

and consequently there exist $0<a<b<c$ such that

$$
\bar{P}(0)>c>b>\bar{P}\left(x_{1}\right) \geqslant \bar{P}\left(x_{2}\right)>a>0 .
$$

By the nature of the construction of $\bar{P}$, there exists a subsequence $\left\{\Delta_{i}\right\}$ and an integer $N$ such that, for all $i>N$,

$$
P_{\Delta_{i}}(0)>c \quad \text { and } \quad P_{\Delta_{i}}\left(x_{1}\right), P_{\Delta_{i}}\left(x_{2}\right) \in(a, b) .
$$

If the monopolist initially charges the price $c$ then, for $i>N$ consumer 0 accepts in the equilibrium associated with $A_{i}$. From the utility maximization of consumer 0 , at least $t$ units of time must pass before the price falls to $b$, where $t$ is defined by

$$
c=\left[1-e^{-r t}\right] f(0)+e^{-r t} b .
$$


Since at least $t$ units of time must pass between prices $c$ and $b$ after $c$ is charged, the profit left in the market for the monopolist facing $P_{\Delta_{i}} . i>N$, when he follows the equilibrium strategy, is at most

$$
\int_{q^{i}(c)}^{x_{1}} P_{\Delta_{i}}+e^{-r t} R^{i}\left(x_{1}\right)
$$

where $q^{i}(c)$ is the state of the market after $c$ is charged, and $R^{i}\left(x_{1}\right)$ is the present value of the monopolist's profits beginning from state $x_{1}$-both according to the equilibrium associated with $\Delta_{i}$.

We next observe that there exist $\hat{t}<t / 2$ and an integer $I>N$ such that

$$
\left|e^{-r \hat{t}} \sum_{j=1}^{K} P_{\Delta_{l}}\left(h_{j}\right) \mu-\int_{q^{i}(c)}^{x_{t}} P_{\Delta_{l}}\right|<a\left[x_{2}-x_{1}\right]\left[e^{-r t / 2}-e^{-r t}\right],
$$

where $K$ is the largest integer that does not exceed $\hat{t} / \Delta_{i}, h_{0}=q^{i}(c), h_{K}=x_{1}$, the $h_{j}$ 's are equally spaced, and $\mu=\left[x_{1}-q^{i}(c)\right] / K$. (This is little more than the statement that the integral of the uniformly bounded, left-continuous function $P_{A_{l}}$ can be approximated uniformly by Riemann sums; for, the right side of (II) is independent of $\hat{t}$ and $i, e^{-r \hat{t}}$ is close to 1 for $\hat{t}$ small, and $K$ is large for $\hat{t}$ fixed and $i$ large.)

We are now able to construct a plan that yields the monopolist more profit than he obtains from the prescribed bahavior in the equilibrium associated with $A_{I}$ after $c$ is charged, and thereby obtain a contradiction. This is achieved by having the monopolist spend $\hat{t}$ units of time getting from $q^{I}(c)$ to $x_{1}$ and then following the strategy prescribed in the equilibrium associated with $\Delta_{I}$. According to (II), his profit will then be at least

$$
\int_{q^{I}(c)}^{x_{1}} P_{d_{l}}-a\left[x_{2}-x_{1}\right]\left[e^{-r t / 2}-e^{-r t}\right]+e^{-r i} R^{l}\left(x_{1}\right) .
$$

But since $\hat{t}<t / 2$ and $R^{\prime}\left(x_{1}\right) \geqslant a\left[x_{2}-x_{1}\right]$, the profit from this plan will exceed the bound on the profit given in (I) for the hypothesized equilibrium strategy.

To get a contradiction for the case that $\bar{P}$ is discontinuous, observe first that $\bar{P}$ is continuous at 1 , so if $x$ is a point of discontinuity then $x<1$. $^{19}$ Now choose $\alpha$ so that $p_{h}>p_{l}$, where

$$
p_{h}=\lim _{q \uparrow x} \bar{P}(q)-\alpha \quad \text { and } \quad p_{l}=\lim _{q \downarrow x} \bar{P}(q)+\alpha .
$$

${ }^{19}$ The continuity of $\bar{P}$ at 1 follows from the fact that $f$ is left-continuous, $\bar{P}(1)=f(1)$ and $\bar{P}(q) \leqslant f(q)$ for all $q \in \mathscr{Q} \subset[0,1]$. 
The existence of such an $\alpha$ is guaranteed by the fact that $\bar{P}$ is non-increasing and $x$ is a point of discontinuity. Observe that since $\bar{P}(q) \leqslant f(q)$ for all $q \in \mathscr{Q}$ and $f$ is left-continuous, there exists $\eta>0$ such that $x+\eta / 2<1$ and $p_{h}<f(x-\eta)$. Hence, there exists $t>0$ that solves

$$
f(x \quad \eta) \quad p_{h}=\left[\begin{array}{ll}
f(x-\eta) & p_{l}
\end{array}\right] e^{-r t} .
$$

By utility maximization of consumers, in any equilibrium if a consumer with a valuation no greater than $f(x-\eta)$ purchases at price $p_{h}$, then at least a duration $t$ must pass before the price falls to $p_{l}$.

For all $\varepsilon \in(0, \eta)$ choose $q_{\varepsilon}^{h} \in(x-\varepsilon / 2, x) \cap \mathscr{Q}$ and $q_{\varepsilon}^{l} \in(x, x+\varepsilon / 2) \cap \mathscr{Q}$. Let $\left\{\Delta_{\varepsilon j}\right\}_{j=0}^{\infty}$ be a subsequence of $\left\{\Delta_{i}\right\}_{i=0}^{\infty}$ such that

$$
\begin{aligned}
& \lim _{j \rightarrow \infty} P_{\Delta_{\varepsilon j}}\left(q_{\varepsilon}^{h}=\bar{P}\left(q_{\varepsilon}^{h}\right),\right. \\
& \lim _{j \rightarrow \infty} P_{\Delta_{\varepsilon j}}\left(q_{\varepsilon}^{l}\right)=\bar{P}\left(q_{\varepsilon}^{l}\right) .
\end{aligned}
$$

The existence of such a sequence is guaranteed by the construction of $\bar{P}$. Pick $n$ such that

$$
P_{\Delta_{s n}}\left(q_{\varepsilon}^{h}\right)>p_{h} \quad \text { and } \quad P_{\Delta_{k n}}\left(q_{\varepsilon}^{l}\right)>p_{l} .
$$

Suppose that $p_{h}$ is charged in the initial period. Since $P_{A_{z n}}\left(q_{\varepsilon}^{h}\right)>p_{h}$, by the fashion in which $P_{\Delta_{s n}}$ defines the strategies of consumers, all consumers $q \leqslant q_{\varepsilon}^{h}$ buy at this price. Observe that $q_{\varepsilon}^{h}>x-\eta$ when $\varepsilon>\eta$; hence, if price $p_{h}$ is charged in the initial period then there will be consumers with valuations no greater than $f(x-\eta)$ that buy, and therefore at least a duration $t$ must elapse before the price falls below $p_{l}$. In other words, there will be at least $t / \Delta_{\varepsilon n}$ price offers before a price below $p_{l}$ can be charged. Let $p_{1}, p_{2}, \ldots, p_{m}$ be the prices above $p_{l}$ that follow $p_{h}$ according to the equilibrium. Define

$$
\begin{aligned}
& a_{0}=p_{h} \\
& a_{i}=p_{h}-i \frac{2 \Delta_{\varepsilon n}}{t}\left[p_{h}-p_{l}\right], \quad i=1, \ldots, \frac{t}{2 \Delta_{\varepsilon n}} .
\end{aligned}
$$

Define the sequence $p_{1}^{\prime}, \ldots, p_{m}^{\prime}$ by $p_{1}^{\prime}=a_{k_{1}}$, where

$$
k_{1}=\inf \left\{r \geqslant 1 \mid \exists p_{j} \in\left[a_{r}, a_{r-1}\right]\right\},
$$

and $p_{i}^{\prime}=a_{k_{i}}$, where

$$
k_{i}=\inf \left\{r \geqslant k_{i-1}+1 \mid \exists p_{j} \in\left[a_{r}, a_{r-1}\right]\right\}
$$


if the inf is over a nonempty set and $k_{i}=t / 2 A_{2 n}$ otherwise ${ }^{20}$ We observe the following points:

First, $m \leqslant t / 2 \Delta_{2 n}$. Hence if the time interval between offers is $\Delta_{z n}$ then the $m$ offers $p_{1}^{\prime}, \ldots, p_{m}^{\prime}$ can be made in $t / 2$ amount of time.

Second, after an initial period in which the price $p_{n}$ is charged, if the monopolist charges the sequence of prices $p_{1}^{\prime}, \ldots, p_{m}^{\prime}$ rather than the sequence $p_{1}, \ldots, p_{\bar{m}}$ then any consumer $q \in[x-\varepsilon / 2, x+\varepsilon / 2]$ that is willing to buy at some price $p_{i}$ is also willing to buy at some price $p_{j}^{\prime}$, where $j \leqslant i$ and $p_{j}^{\prime} \geqslant p_{i}-2 A_{\varepsilon n} / t$. Hence the monopolist charging the sequence of prices $p_{1}^{\prime}, \ldots, p_{m}^{\prime}$ rather than $p_{1}, \ldots, p_{\bar{m}}$ does not lose time and loses at most $\left[p_{h}-p_{l}\right] 2 \Delta_{\varepsilon n} / t$ on each sale.

Third, if $R_{1}^{\varepsilon}$ is the return to the monopolist from the strategy that has him charging $p_{h}, p_{1}, \ldots, p_{\bar{m}}$ in the first $\bar{m}$ periods and playing optimally thereafter, and if $R_{2}^{\epsilon}$ is the return to the monopolist charging $p_{h}, p_{1}^{\prime}, \ldots, p_{m}^{\prime}$ in the first $m$ periods and then continuing optimally, then since $\bar{m} \Delta_{\varepsilon n} \geqslant t$ and $m \Delta_{\varepsilon n} \leqslant t / 2$ we have

$$
R_{2}^{\varepsilon}-R_{1}^{\varepsilon} \geqslant\left[e^{-r t / 2}-e^{-r t}\right] R^{\varepsilon}(x+\varepsilon / 2)-\frac{2 \Delta_{\varepsilon n}}{t} \varepsilon\left(p_{h}-p_{l}\right],
$$

where $R^{\varepsilon}(x+\varepsilon / 2)$ is the monopolist's maximal return after state $x+\varepsilon / 2$ in the equilibrium associated with $P_{\Lambda_{E n}}$. Hence,

$$
R_{2}^{s}-R_{1}^{s} \geqslant\left[e^{-r t / 2}-e^{-r t}\right]\left[1-e^{-r \Delta_{e n}}\right] M-\left(2 \Delta_{e n} / t\right) \&\left[p_{h}-p_{I}\right],
$$

where

$$
M=\max _{y} f(y)[y-x-\eta / 2],
$$

using the fact that

$$
P_{\Delta_{f n}} \geqslant\left[1-e^{-r \Delta_{\varepsilon n}}\right] f \quad \text { and } \quad \varepsilon<\eta .
$$

Let

$$
\begin{aligned}
& A=\left[e^{-r t / 2}-e^{-r t}\right] M, \\
& B=2\left[p_{h}-p_{l}\right] / t .
\end{aligned}
$$

Then $A, B>0$ and $A$ and $B$ are independent of $\varepsilon$ and $A_{\varepsilon n}$; furthermore,

$$
R_{2}^{\varepsilon}-R_{1}^{\varepsilon} \geqslant\left[1-e^{-r A_{\varepsilon n}}\right]\left[A-B \varepsilon \frac{\Delta_{\varepsilon n}}{1-e^{-r A_{\varepsilon n}}}\right]
$$

${ }^{20}$ An obvious adjustment is required if $t / 2 A_{g n}$ is not an integer. 
Since $1-e^{-r \Delta_{s n}}>0$ and the function $g(\Delta) \equiv \Delta /\left[1-e^{-r \Delta}\right]$ is bounded on the interval $\left(0, A_{1}\right]$ for sufficiently small $\varepsilon>0$, we have $R_{2}^{\varepsilon}-R_{1}^{\varepsilon}>0$. But this contradicts the fact that the sequence of prices $p_{1}, \ldots, p_{\bar{m}}$ were optimal for the monopolist after charging the price $p_{h}$ in the initial period.

\section{RFFERENCES}

1. J. I. BuLow, Durable-goods monopolists, J. Polit. Econ. 90 (1982), 314-332.

2. R. H. CoAsE, Durability and monopoly, J. Law Econ. 15 (1972), 143-149.

3. P. C. Cramton, "The Role of Time and Information in Bargaining," Ph.D. dissertation, Stanford University, 1984.

4. D. Fudenberg, D. Levine, and J. Tirole, Infinite horizon models of bargaining with one-sided incomplete information, in "Bargaining with Incomplete Information" (Alvin Roth, Ed.), London/New York, Cambridge Univ. Press, pp.73-98, 1985.

5. S. J. Grossman and M. Perry, Sequential bargaining under asymmetric information, working paper, University of Chicago, December 1984.

6. F. Gul, "Two Essays on the Bargaining Foundations of Value Theory," Ph.D. dissertation, Princeton University, 1986.

7. Charles Kahn, The durable goods monopolist and consistency with increasing costs. Econometrica 54 (1986), 275-294.

8. A. Rubinstein, Perfect equilibrium in a bargaining model, Econometrica 50 (1982), $97-110$.

9. A. RUBlNSTEIN, A bargaining model with incomplete information about preferences, Econometrica 53 (1985), 1151-1172.

10. J. Sobel AND I. Takahashi, A multi-stagc model of bargaining, Rev. Econ. Stud. 50 (1983), 411-426.

11. N. L. StokeY, Rational expectations and durable goods pricing, Bell J. Econ. 12 (1982), $112-128$. 